\title{
Stiffness Regulates Intestinal Stem Cell Fate
}

Shijie He ${ }^{1,2,3,4^{*}}$, Peng Lei ${ }^{1,2,3,4^{*}}$, Wenying Kang ${ }^{5}$, Priscilla Cheung ${ }^{4,6}$, Tao $\mathrm{Xu}^{4,7}$, Miyeko Mana ${ }^{8}$, Chan Young Park ${ }^{9}$, Hongyan Wang ${ }^{1,4}$, Shinya Imada ${ }^{8}$, Jacquelyn O. Russell ${ }^{4,6}$, Jianxun Wang ${ }^{1,2,3,4}$, Ruizhi Wang ${ }^{10}$, Ziheng Zhou ${ }^{1,2,3,4}$, Kashish Chetal ${ }^{4,11}$, Eric Stas ${ }^{4,12}$, Vidisha Mohad ${ }^{1,4}$, Marianna Halasi ${ }^{2,3,4}$, Peter Bruun-Rasmussen ${ }^{13}$, Ruslan I. Sadreyev $^{4,11,14}$, Irit Adini ${ }^{2,3,4}$, Richard A. Hodin ${ }^{1,4}$, Yanhang Zhang ${ }^{10}$, David T. Breault ${ }^{4,12,15}$, Fernando D. Camargo $^{4,6,15}$, Ömer H. Yilmaz ${ }^{8}$, Jeffrey J. Fredberg ${ }^{9}$, and Nima Saeidi ${ }^{1,2,3,4,15 \#}$

${ }^{1}$ Division of Gastrointestinal and Oncologic Surgery, Department of Surgery, Massachusetts General Hospital, Boston, MA 02114, USA

$10{ }^{2}$ Center for Engineering in Medicine and Surgery, Department of Surgery, Massachusetts General Hospital, Boston, 11 MA 02114, USA

$12 \quad{ }^{3}$ Shriners Hospital for Children - Boston, MA 02114, USA

$13 \quad{ }^{4}$ Harvard Medical School, Boston, MA 02115, USA

$14{ }^{5}$ Deparment of Otolaryngology- Head and Neck Surgery, Stanford Medical School, CA 94305, USA

${ }^{6}$ Stem Cell Program and Department of Hematology/Oncology, Children's Hospital, Boston, MA 02115, USA

${ }^{7}$ Section on Pathophysiology and Molecular Pharmacology, Joslin Diabetes Center, Boston, MA 02115, USA

${ }^{8}$ Koch Institute for Integrative Cancer Research, Massachusetts Institute of Technology, Cambridge, MA 02142, USA

${ }^{9}$ Department of Environmental Health, Harvard T.H. Chan School of Public Health, Boston, MA 02115, USA

${ }^{10}$ Department of Mechanical Engineering, Boston University, Boston, MA 02215, USA

${ }^{11}$ Department of Molecular Biology, Massachusetts General Hospital, Boston, MA 02114, USA

${ }^{12}$ Division of Endocrinology, Boston Children's Hospital, Boston, MA 02115, USA

${ }^{13}$ Department of Clinical Immunology, Rigshospitalet, Copenhagen University Hospital, DK-2200, Copenhagen, Denmark

${ }^{14}$ Department of Pathology, Massachusetts General Hospital

${ }^{15}$ Harvard Stem Cell Institute, Cambridge, MA 02138, USA

27

* Shijie He and Peng Lei contributed equally. 


\section{Summary}

Does fibrotic gut stiffening caused by inflammatory bowel diseases (IBD) direct the fate of intestinal stem cells (ISCs)? To address this question we first developed a novel long-term culture of quasi-3D gut organoids plated on hydrogel matrix of varying stiffness. Stiffening from $0.6 \mathrm{kPa}$ to $9.6 \mathrm{kPa}$ significantly reduces $\mathrm{Lgr5}^{\text {high }} \mathrm{ISCs}$ and $\mathrm{Ki}^{+} 7^{+}$progenitor cells while promoting their differentiation towards goblet cells. These stiffness-driven events are attributable to YAP nuclear translocation. Matrix stiffening also extends the expression of the stemness marker Olfactomedin 4 (Olfm4) into villus-like regions, mediated by cytoplasmic YAP. We next used single-cell RNA sequencing to generate for the first time the stiffness-regulated transcriptional signatures of ISCs and their differentiated counterparts. These signatures confirm the impact of stiffening on ISC fate and additionally suggest a stiffening-induced switch in metabolic phenotype, from oxidative phosphorylation to glycolysis. Finally, we used colon samples from IBD patients as well as chronic colitis murine models to confirm the in vivo stiffening-induced epithelial deterioration similar to that observed in vitro. Together, these results demonstrate stiffness-dependent ISC reprograming wherein YAP nuclear translocation diminishes ISCs and $\mathrm{Ki}^{+} 7^{+}$progenitors and drives their differentiation towards goblet cells, suggesting stiffening as potential target to mitigate gut epithelial deterioration during IBD.

Upon migrating on the soft basement matrix $(\mathrm{BM})$ from the bottom of the crypt to the tip of the villus, intestinal stem cells (ISCs) differentiate to diverse types of gut epithelial cells, including Paneth cells, goblet cells, enteroendocrine cells (EECs), tuft cells, microfold (M) cells and enterocytes ${ }^{1}$. Inflammatory bowel disease (IBD), which includes ulcerative colitis (UC) and Crohn's disease (CD), is associated with 
the deterioration of gut epithelium, including reduction of $\mathrm{ISCs}^{2}$ and increase of $\mathrm{M}$ cells in UC ${ }^{3}$. Furthermore, due to the excessive secretion of collagen, the BM stiffens ${ }^{4-6}$. It has been demonstrated that the stiffness of the BM can regulate the differentiation of mesenchymal stem cells, the progenitor cells of central nervous system and pancreatic progenitors ${ }^{7-9}$. Yet, it is unclear how the BM stiffening in IBD impacts the fate of ISCs and their differentiation, and contributes to the epithelium deterioration.

\section{Quasi-3D gut organoids cultured on soft hydrogel matrix}

To investigate the impact of BM stiffening on the differentiation of ISCs, we developed a platform for culturing quasi-3D gut organoids on top of soft polyacrylamide-hydrogel matrix (Fig. 1A). ISCs and their crypts were harvested from mice and seeded on the hydrogel matrix. Lgr5-EGFPIRES-creERT2 mice were used to track Lgr5 ${ }^{+}$ISCs (Extended Data Fig. 1). As the organoids grew, the soft hydrogel surface buckled $\left(0.6 \mathrm{kPa}\right.$, matching that of a healthy $\left.\mathrm{BM}^{6}\right)$, forming a quasi-3D structure that mimicked the invagination of the in vivo crypts ( Fig. 1A, and 3D confocal imaging in Fig. 1L). The crypt-like regions were densely populated by the ISCs intermixed with the large, optically dark, UEA ${ }^{+}$Paneth cells (Fig. 1B). The peripheries of the crypts were surrounded by the transit-amplifying (TA) progenitor cells with strong Ki67 expression, and Ki67 was also weakly expressed in Lgr $5^{\text {high }}$ ISCs (Fig. 1B and Extended Data Fig. 2A). The villus-like regions were populated by Villin ${ }^{+}$enterocytes (Fig. 1B), Muc2 ${ }^{+}$goblet cells, and Chromogranin-A ${ }^{+}$(Chro-A) EECs (Extended Data Fig. 2B). Notably, the villus-like regions also exhibited a turnover rate of approximately 3 days (Extended Data Fig. 3), similar to that observed in vivo. By culturing these quasi-3D gut organoids on the hydrogel matrix of varying stiffness, we analyzed the impact of stiffness on the fate of ISCs and their preference of differentiation directions. 
A

Quasi-3D gut organoids

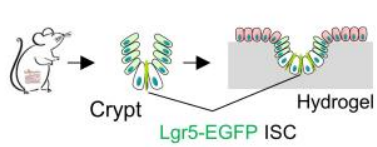

C

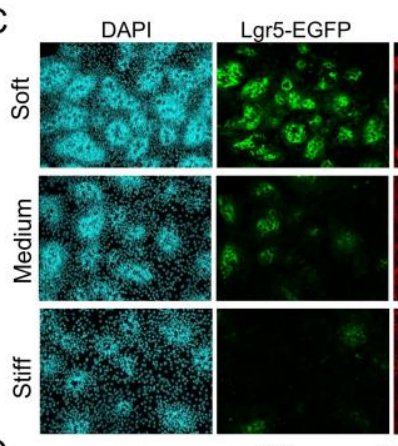

D

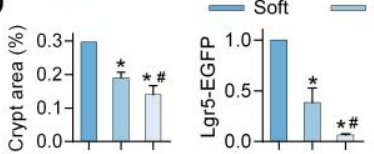

$\mathrm{E}$
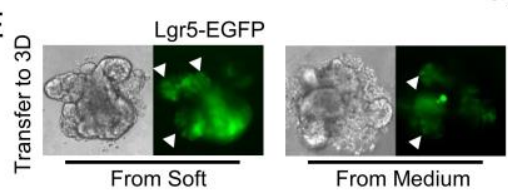

From Medium

F

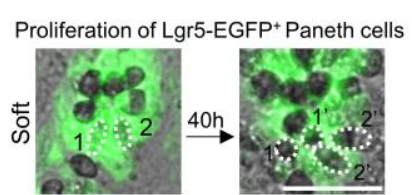

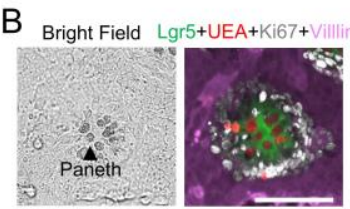
Olfm4
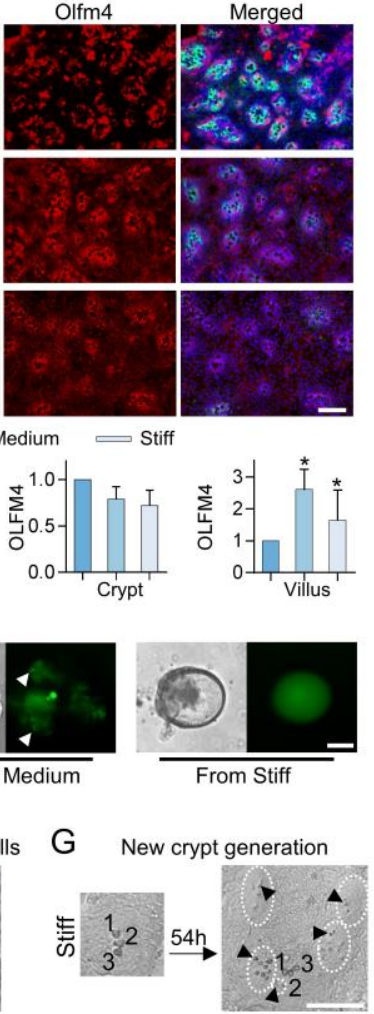
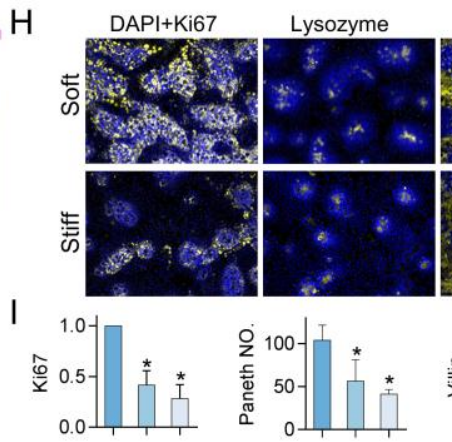

$\mathrm{J}$
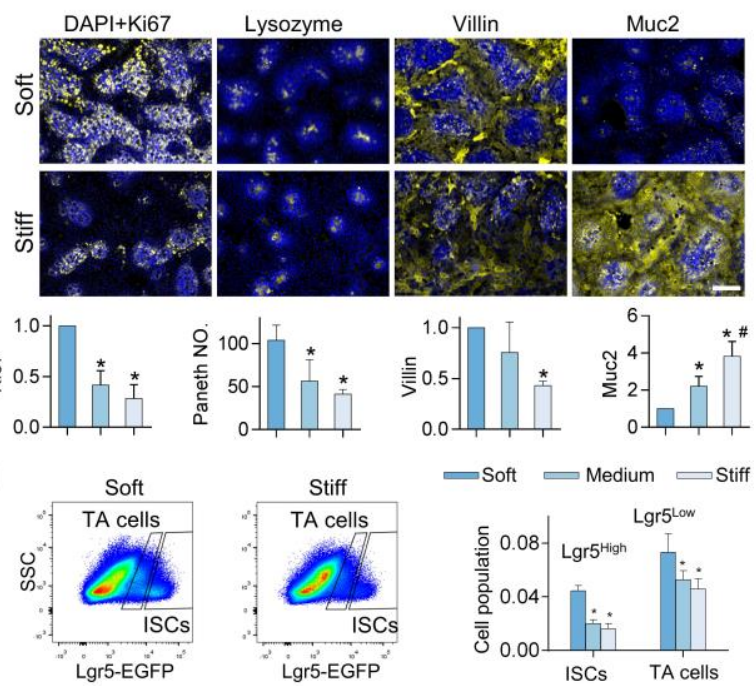

K
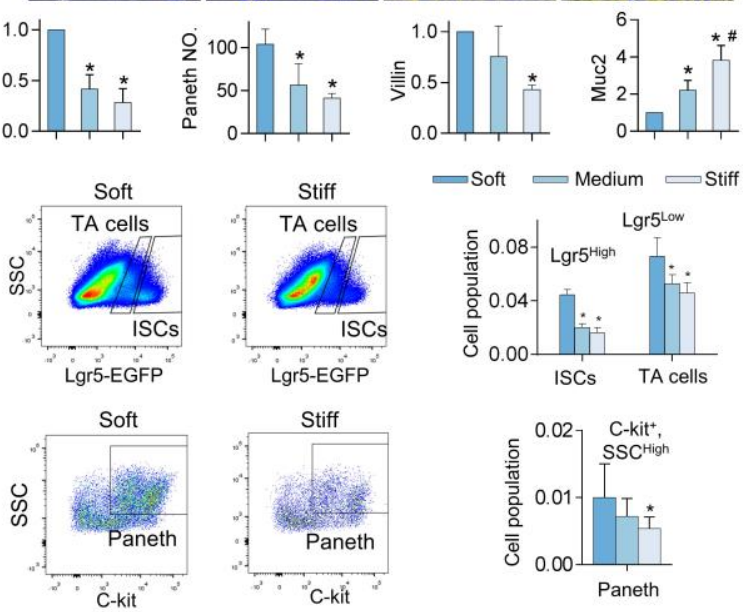

$\mathrm{L}$
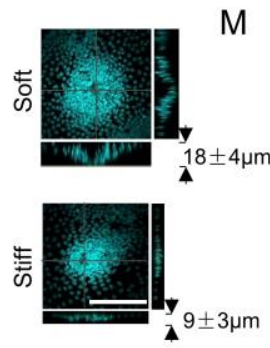

Figure 1. Stiffness determines the fate of ISCs. (A) Illustration of the experimental system. (B) Lgr5-EGFP ${ }^{+}$ISCs $_{\text {were intermixed with }}$ the optically dark UEA ${ }^{+}$Paneth cells, which were surrounded by Ki67+ TA cells in the crypt-like regions. The villus-like regions were populated by Villin ${ }^{+}$differentiated cells. (C) The matrix stiffening from soft $(0.6 \mathrm{kPa})$ to medium $(2.4 \mathrm{kPa})$ to stiff $(9.6 \mathrm{kPa})$ reduced the size of the crypt-like regions with the dense nuclei and decreased the expression of Lgr5. Stiffening extended Olfm4 into the villus-like regions. (D) Quantification of the fluorescent intensity per unit area of crypt / villus regions. The crypt and villus regions were segmented using customized code based on DAPI intensity (Method, $n=3-5$ ). (E) The 3D organoids derived from the soft and medium matrix budded with Lgr5-EGFP ${ }^{+}$ISCs (white arrows). The 3D organoids derived from the stiff matrix grew more like Lgr5-EGFP ${ }^{-}$cysts. $n=3$. (F) Lgr5-EGFP ${ }^{+}$ ISCs ( 1 and 2) differentiated into two Paneth cells ( 1 ' and 2') on the soft matrix (Movie S1, $n=3$ ). (G) On the stiff matrix cells in the villuslike regions differentiated into Paneth cells (black arrows), which was followed by the new crypt generation (white dashed line, Movie S2, $n=3)$. (H) The stiffening decreased the expression of Ki67, Lysozyme and Villin, but increased Muc2, as quantified via fluorescent intensity (I, $n=3-5)$. ( $\mathrm{J}$ and K) Flow cytometry analysis showed that stiffening decreased Lgr $5^{\text {high }}$ ISCs, Lgr5 $5^{\text {low }}$ TA cells, and Paneth cells ( $n=3$ ). (L) $3 \mathrm{D}$ confocal imaging showed that the stiffening significantly inhibited the crypt invagination $(P<0.05, n=3)$. (M) A schematic summarizes the impact of stiffening on all cell types. 'P', Panetch cell. Scale bar, $100 \mu \mathrm{m}$. * V.S. Soft and \# V.S. Medium, $P<0.05$ (Student's $t$-test).

\section{Stiffening reduces the number of $\mathrm{Lgr}^{+}$ISCs and promotes their differentiation into}

\section{immature enterocyte-goblet cells (IEGCs)}


Increasing the matrix stiffness from soft $\left(0.6 \mathrm{kPa}\right.$, matching that of a healthy $\left.\mathrm{BM}^{6}\right)$ to medium $(2.4$

$\mathrm{kPa})$ to stiff $\left(9.6 \mathrm{kPa}\right.$, matching that of an inflamed $\left.\mathrm{BM}^{6}\right)$ gradually decreased the crypts surface area and reduced the number of Lgr5-EGFP ${ }^{+}$ISCs (Fig. 1C and 10-days live-cell imaging for Lgr5-EGFP in Extended Data Fig. 4A). To further verify the impact of stiffness on ISCs, after 11 days of culture, the cells were detached from the hydrogel matrix and transferred to the inside of Matrigel ${ }^{\circledR}$ to grow 3D organoids (Method). The 3D organoids from the soft or medium matrix budded to form the crypt regions with the Lgr5-EGFP+ ISCs, but those from the stiff matrix grew more like cysts with a significantly smaller number of buddings (Fig. 1E and quantified in Extended Data Fig. 4B), confirming the loss of stemness on the stiff matrix.

In addition to Lgr5, the expression and distribution of another stem cell marker, Olfactomedin 4 (Olfm4), exhibited strong correlation with the stiffness. On the soft matrix, Olfm $4^{+}$cells were concentrated in the periphery of the crypt-like regions (Fig. 1C and 1D). Upon increasing stiffness, Olfm $4^{+}$cells became interspersed throughout the crypt region and replaced the $\mathrm{Lgr}^{+}$ISCs to directly border with Paneth cells (Figs. 1C and Extended Data Fig. 5). Notably, on the stiff matrix, Olfm4 expression extended into the villus-like regions.

Live-cell imaging on the soft matrix showed that Lgr5-EGFP ${ }^{+}$ISCs divided and differentiated into large and optically dark Paneth cells (Fig. 1F and Movie S1) which, in turn, contributed to the maintenance of ISC niche and stemness ${ }^{10}$. In contrast, Lgr5-EGFP ${ }^{+}$ISCs greatly diminished on the stiff matrix (Fig. 1C and Extended Data Fig. 4A). However, on the stiff matrix the cells in the villus-like regions differentiated into Paneth cells and ultimately generated ectopic, new crypt-like regions (Fig. 1G and Movie S2). The incidence of these ectopic crypts was approximately three-fold higher on the stiff matrix 
compared to the soft matrix (Extended Data Fig. 4C and Movie S3).

Stiffening also altered the proportion of differentiated cells. Stiffening diminished $\mathrm{Ki} 67^{+}$proliferating progenitor cells and Lysozyme ${ }^{+}$Paneth cells, as well as the expression of the enterocyte markers, Villin and Alpi ${ }^{11}$ (Fig. 1H and I, and Alpi in Extended Data Fig. 6). The EEC marker - Chro-A was also decreased by stiffening (Extended Data Fig. 6). In contrast, markers of secretory progenitor cells- Dl11 ${ }^{12}$ and goblet cells- Muc2 were increased (Fig. 1H and I, and Dl11 in Extended Data Fig. 6). Notably, on the stiff matrix a new cell type emerged in the villus-like regions that co-expressed the markers for enterocytes, goblet cells, and stem cells (i.e., Villin, Muc2, and Olfm4, Fig. 1C, 1H and Extended Data Fig. 7). We named this new cell type the immature enterocyte-goblet cell (IEGC), where 'immature' refers to the coexpression of the different cell type markers. Flow cytometry analysis (Method) confirmed the reduction of ISCs (Lgr5-EGFP $\left.{ }^{\text {high }}\right)$, TA cells (Lgr5-EGFP ${ }^{\text {low }}$ ) and Paneth cells $\left(C D 24^{\text {high }}\right.$, C-kit $^{+}$and SSC $\left.{ }^{\text {high }}\right){ }^{13,14}$ on the stiff matrix (Fig. 1J and K). Interestingly, 3D confocal imaging showed that the depth of the cryptlike regions was two-fold greater on the soft matrix compared to the stiff matrix (Fig. 1L).

The schematic in Fig. 1M summarizes the impact of stiffening on the various cell types: in the interior of crypt-like regions, stiffening led to the loss of $\mathrm{Lgr}^{5^{\text {high }}}$ ISCs cells and the appearance of Olfm $4^{+}$stem cells which were directly adjacent to Paneth cells. It also reduced $\mathrm{Lgr} 5^{\text {low }}, \mathrm{Ki} 67^{+} \mathrm{TA}$ progenitor cells. In the villus-like regions, stiffening led to the replacement of the Villin ${ }^{+}$mature enterocytes by Muc2 ${ }^{+}, \mathrm{Villin}^{+}$, and Olfm $4^{+}$IEGCs.

\section{The impacts of stiffness on ISC fate are YAP-dependent}

Matrix stiffening stimulated YAP expression (Fig. 2A and 2D) and promoted YAP nuclear translocation (Fig. 2A and Extended Data Fig. 8). Lgr $5^{\text {high }}$ ISCs were YAP negative, and YAP expression 
131 was inversely correlated with Lgr5 expression (Fig. 2A). To better distinguish the expression patterns of 132 cytoplasmic YAP (cyto-YAP) and nuclear YAP (nuc-YAP), we performed immunostaining for non133 phosphorylated YAP and the Ser 127 phosphorylated YAP, since YAP nuclear translocation is negatively 134 regulated by YAP phosphorylation ${ }^{15}$. The non-phosphorylated YAP expression was uniformly increased across both the crypt- and villus-like regions on stiff matrix and showed pronounced nuclear localization (referred as nuc-YAP for simplicity in Fig. 2B, quantified in 2D). In contrast, phosphorylated YAP that is primarily cytoplasmic exhibited strong region-dependent expression, i.e., it was decreased by the stiffening in the crypt-like regions, but increased in the villus-like regions (referred as cyto-YAP in Fig. 2C, quantified in 2D). To assess the relationship between YAP expression and the ISC differentiation trajectory, we counterstained total YAP with the markers of the differentiated cells. Proliferating cell marker (Ki67, Extended Data Fig. 9) and stem cell marker (Olfm4, Fig. 2E and crypt-like region in

142 Extended Data Fig. 10) were both positively correlated with cyto-YAP, whereas goblet cell marker was 143 positively correlated with nuc-YAP (Muc2, Fig. 2F). The Villin ${ }^{+}$enterocytes on the soft matrix were YAP 144 negative, and the Villin expression tended to decrease when YAP showed nuclear localization on the stiff 145 matrix (Fig. 2G). Notably, the epidermal growth factor receptor (Egfr) which is involved in the process of 146 inflammation and cancer ${ }^{16}$ was positively correlated with cyto-YAP (Extended Data Fig. 11), similar to 147 the stem cell marker, Olfm4. 

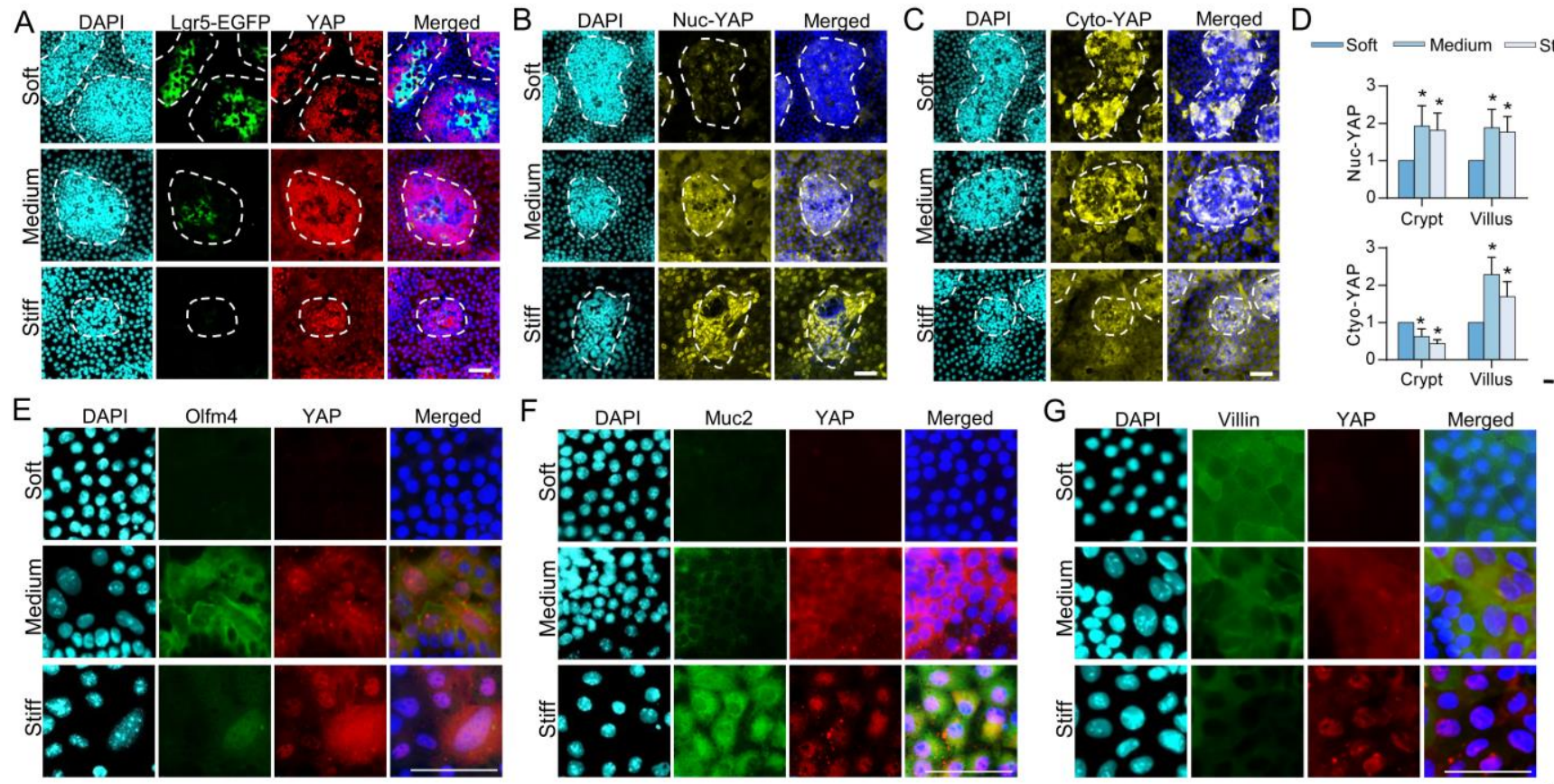

$\mathrm{H}$
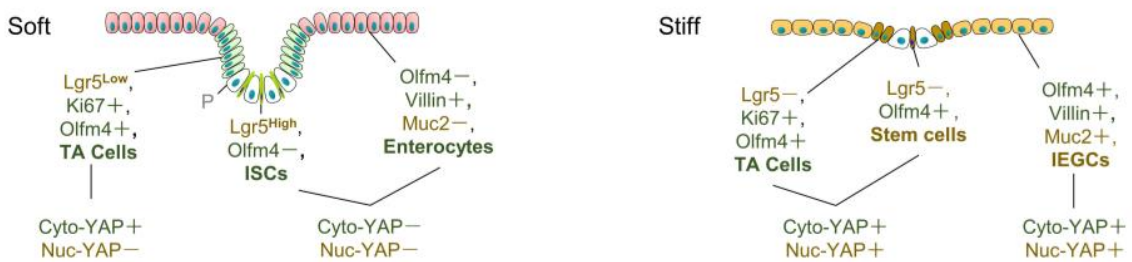

149

150

151

152

153

154

155

156

Figure 2. Stiffness regulates the fate of ISCs via YAP (A) Lgr5-EGFPhigh ISCs were YAP $^{-}$and disappeared when YAP was nuclear colocalized on the stiff matrix. The white dashed lines trace the crypt-like regions $(n=3)$. (B) The non-phosphorylated nuclear (nuc-) YAP was increased by stiffening and showed clear nuclear co-localization on the stiff matrix $(n=5)$. (C) The Ser 127 phosphorylated cytoplasmic (cyto-) YAP was decreased by stiffening in the crypt-like regions, but increased in the villus-like regions, which were quantified via fluorescent intensity (D, * V.S. Soft, $P<0.05, n=5$ ). (E) Olfm4 was highly expressed together with cyto-YAP ( $n=3$ ). (F) Muc2 was highly

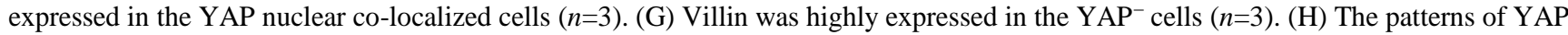
were mapped onto all the cell types that were negatively (Green) or positively (Yellow) correlated with YAP nuclear translocation. Scale bar, $25 \mu \mathrm{m}$.

Based on the expression patterns of the nuc- and cyto-YAP (Fig. 2) we hypothesized that they play divergent roles in regulating the ISC differentiation patterns, where nuc-YAP appears to drive the differentiation into goblet cells, while cyto-YAP drives the differentiation into TA cells, enterocytes, and Olfm $4^{+}$stem cells (Fig. 2H). More specifically, on the soft matrix when the YAP $^{-}$and Lgr5 ${ }^{\text {high }}$ ISCs migrate up, high cyto-YAP expression drives their differentiation into Ki67 ${ }^{+}$TA cells (Extended Data Fig. 
9). Continuously driven by cyto-YAP, the TA cells primarily mature into enterocytes, which lose YAP expression after maturation (Fig. 2G). On the stiff matrix the constitutive expression of YAP causes the loss of Lgr5 ${ }^{\text {high }}$ ISCs (Fig. 2A) and gain of Olfm4 instead (i.e., nuc-YAP ${ }^{+}$, cyto-YAP ${ }^{+}$, and Olfm4 ${ }^{+}$stem cells). These Olfm $4^{+}$stem cells have the potential to simultaneously differentiate into enterocytes driven by cyto-YAP and into goblet cells driven by nuc-YAP, which results in the new cell type, IEGCs (Fig. $2 \mathrm{H})$.

\section{Nuc-YAP and cyto-YAP play divergent roles in determining the fate of ISCs}

To test our hypothesis, transgenic mouse models were employed to knockout $(\mathrm{KO})$ or overexpress $(\mathrm{OE})$ YAP. Verteporfin (VP) was used to suppress YAP nuclear translocation ${ }^{17}$. YAP KO led to the loss of the villus-like regions (Fig. 3A), indicating the indispensability of YAP in the differentiation of ISCs and the generation of villi. The leftover crypt-like regions were enriched with Paneth cells and were negative for nuc-YAP and Muc2, as well as cyto-YAP, Olfm4, Villin, and Egfr (Extended Data Fig. 12). YAP OE induced by doxycycline (DOX) led to the disappearance of the large and dense crypt-like regions on the soft matrix (Fig. 3B), causing a shift towards the stiff matrix-like phenotypes. Conversely, VP administration on the stiff matrix led to the formation of large crypt-like regions (Fig. 3C) and the restoration of Lgr5 expression (Fig. 3D), bestowing soft matrix-like phenotypes.

Comparing the phenotypes between YAP OE and VP administration showed that nuc-YAP was increased in YAP OE but decreased in VP (Fig. 3E and 3G). In contrast, cyto-YAP was consistently increased in both models (Fig. 3F and 3H). Therefore, comparison of these two models enables us to discriminate the functional roles of nuc-YAP and cyto-YAP. Increasing nuc-YAP expression by OE promoted Muc2 (Fig. 3E), whereas decreasing nuc-YAP by VP suppressed Muc2 (Fig. 3G). Meanwhile, 


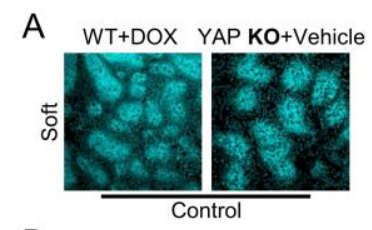

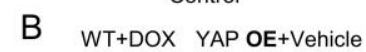

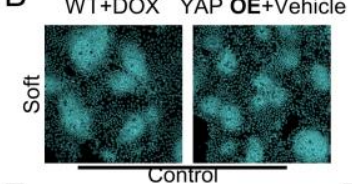

E
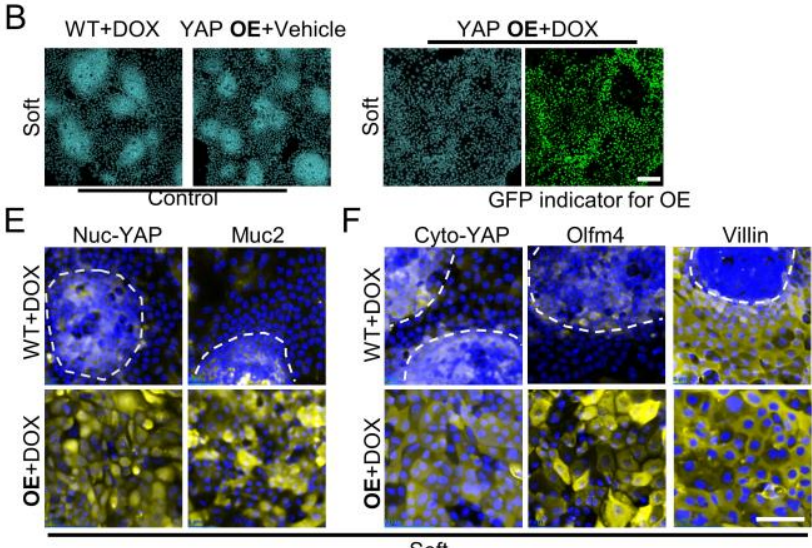

I

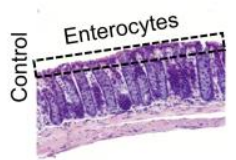

$J$

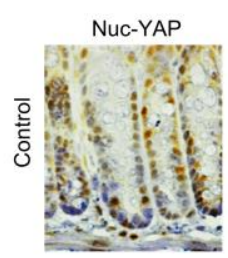

$\mathrm{F}$

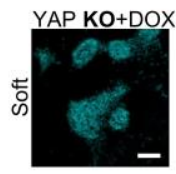

Soft

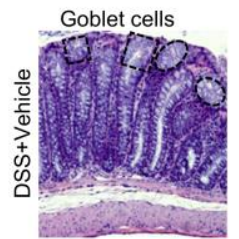

Nuc-YAP

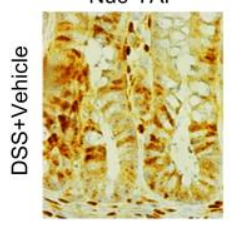

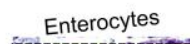

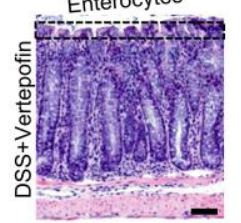

Nuc-YAP

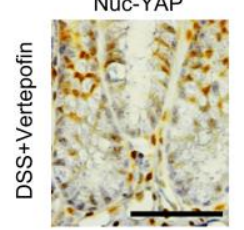

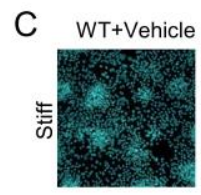

G

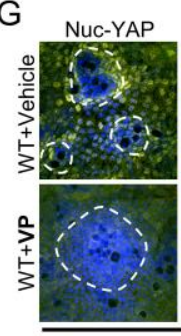

$\mathrm{K}$

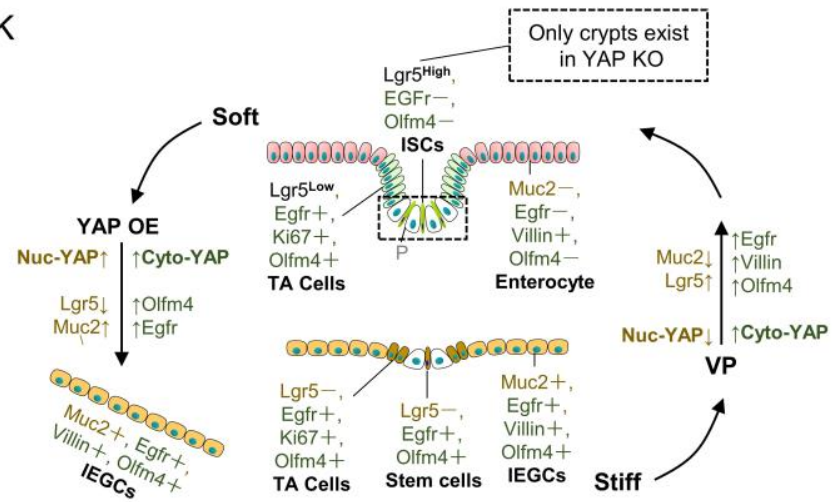

Figure 3. The fate of ISCs was manipulated via YAP knockout (KO), overexpression (OE), and Verteporfin (VP). (A) The villus-like regions vanished in the YAP KO groups. (B) YAP OE led to the loss of the crypt-like regions on the soft matrix. (C and D) VP administration increased the size of the crypt-like regions and resumed the Lgr5-EGFP expression on the stiff matrix. (E) Increase of nuc-YAP via OE augmented Muc2. (F) Increase of cyto-YAP via OE augmented Olfm4. No significant changes of Villin were detected. (G) Decrease of nucYAP via VP suppressed Muc2. (H) Increase of cyto-YAP via VP augmented Olfm4 and Villin. (I) Goblet cells replaced the enterocyte in the colon brush border in DDS-induced colitis group, and VP reversed this replacement. (J) Nuc-YAP was increased in the DDS-induced colitis group, but VP suppressed nuc-YAP. (K) YAP OE transformed the soft-matrix phenotypes into the stiff-matrix phenotypes, and VP did the 
opposite. 'Yellow' indicates the regulation by nuc-YAP. 'Green', the regulation by cyto-YAP. The white dashed lines trace the crypt-like regions. Scale bars in A, B and E, $100 \mu \mathrm{m}$; I and J, $200 \mu \mathrm{m}$; the rest, $25 \mu \mathrm{m}$. $n=3$ for these experiments.

To assess the impact of tissue stiffness and VP in vivo, we administered VP in the dextran sulfate sodium (DSS)-induced chronic colitis mouse model (Method). In the colitis mouse the colon thickened (Fig. 3I and Extended Data Fig. 15A) and stiffened (Extended Data Fig. 15B). Moreover, colitis induced the replacement of enterocytes by goblet cells in the colon brush border, which was reversed by VP administration (Fig. 3I). Mechanistically, similar to the in vitro VP administration, the stiffened colon in the colitis mouse increased nuc-YAP which promoted the differentiation towards to goblet cells, and VP suppressed the stiffening-induced increase of nuc-YAP as well as goblet cell differentiation (Fig. 3I and 3J). The increase of cyto-YAP in the VP treatment group augmented the expression of Olfm4 and Egfr, which is also consistent with the in vitro observation (Extended Data Fig. 15D). Therefore, we demonstrated that YAP is indispensable for the ISC differentiation whereby nuc-YAP drives the differentiation towards Muc2 ${ }^{+}$goblet cells, and cyto-YAP drives the differentiation towards Villin ${ }^{+}$ enterocytes meanwhile promoting the expression of Egfr and Olfm4 (Fig. 3K).

\section{Stiffness-regulated transcriptional signatures}

We generated, for the first time, the stiffness-regulated transcriptional signatures of the ISCs and their differentiated cells using single-cell RNA sequencing (scRNAseq) analysis of our quasi-3D organoids.

The single-cell expression profiles were clustered into thirteen cell groups (Fig. 4A) and the most highly expressed genes of each group were easily distinguishable (Supplementary Table 1). Using the known marker genes ${ }^{18}$, they were identified as various types of gut epithelial cells, matching the in vivo gut epithelium (Figs. 4A and 4B, and Extended Data Fig. 16). The clustering was consistent across both the soft and stiff matrix (Extended Data Fig. 17). Notably, two cell groups were identified as IEGCs which 
224 In fact, Tff3 was upregulated across most of the cells on the stiff matrix (Fig. 4D), which was also 225 confirmed at the protein level (Extended Data Fig. 18). These results suggest a preferential differentiation 226 of ISCs towards goblet cells on the stiff matrix. In addition, the stiffening significantly increased M cells 227 and decreased tuft cells (Fig. 4C). 
A

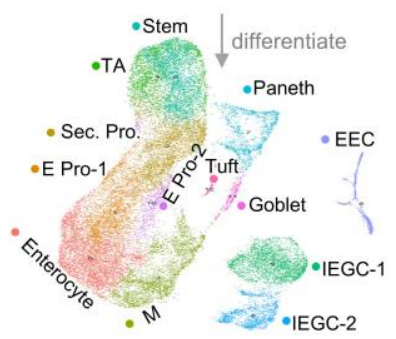

Absorptive

D

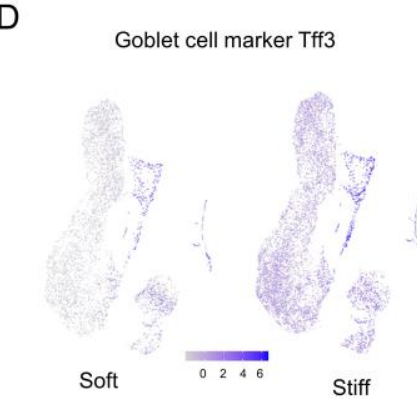

B

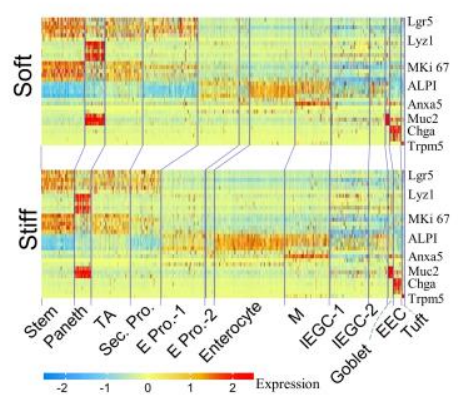

E

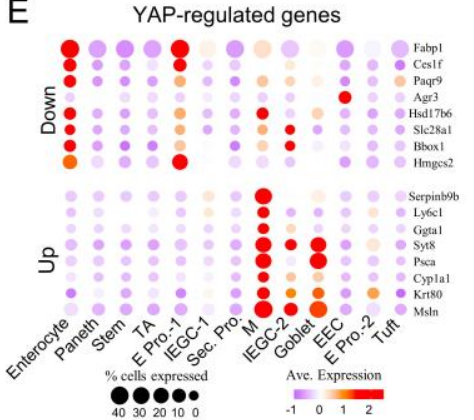

C

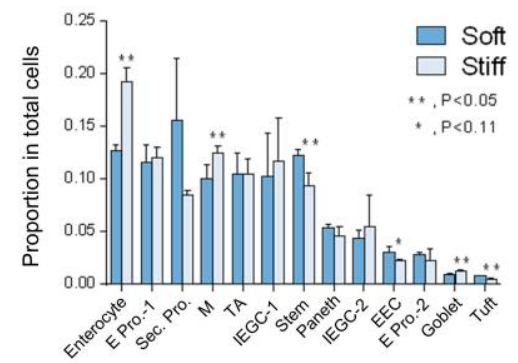

$\mathrm{F}$

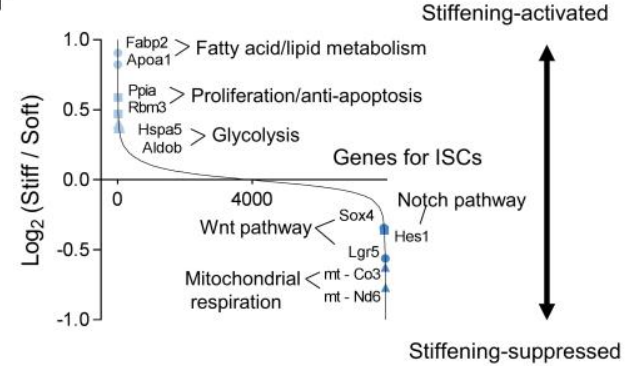

G

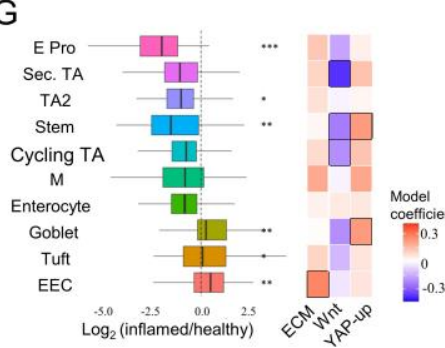

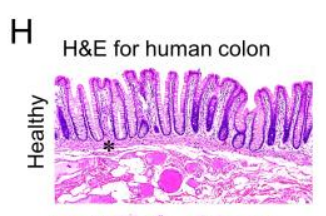
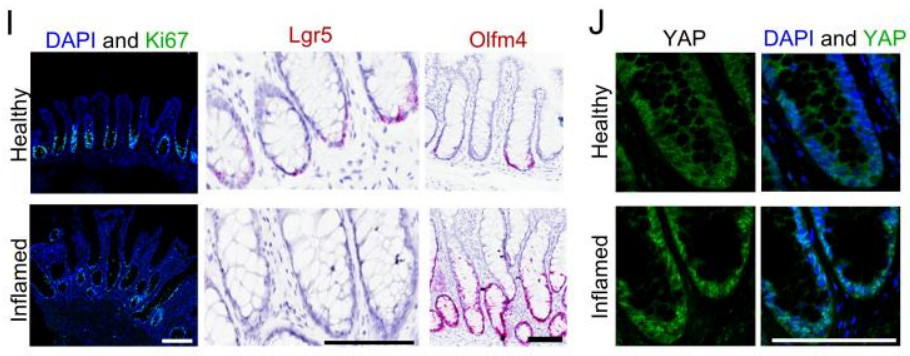

Figure 4. Single cell RNA sequencing and histology from IBD patient. (A) UMAP plot with the cell clusters (marked by color) including ISCs and the differentiated cells. 'Sec', secretory; 'Pro', progenitor; 'E', enterocyte; 'M', microfold. (B) Heat map for marker genes of each cell type (Extended Data Fig. 16 for full version). (C) The proportions of each cell type on the soft and stiff matrix. (D) Expression of Tff3 was higher on the stiff matrix than on the soft matrix. (E) The genes downregulated by YAP were highly expressed in enterocytes and E pro.1; however, the genes upregulated by YAP were highly expressed in goblet cells, IEGCs-1 and M cells. (F) Differential gene expression analysis in ISCs showed that stiffening suppressed both Wnt signaling (e.g., Lgr5 and Sox4 genes), and Notch signaling (e.g., Hes1), and possibly switched metabolic phenotype from mitochondrial respiration (e.g., downregulated mt-Co3 and me-No6) to glycolysis (e.g., upregulated Hspa5 and Aldob). $n=3$ for A-F. (G) Compared to healthy individuals $(n=5)$, relative proportions of cell types in IBD patients $(n=3)$ showed a decrease of ISCs, an increase of goblet cells and a trend towards a decrease of enterocytes. $* P<0.05, * * P<0.01, * * * P<0.001$. Pathway enrichment analysis shows that in IBD patients ECM secretion is activated in EECs, Wnt signaling is suppressed in ISCs and the YAP up-regulated genes are highly expressed in ISCs and goblet cells. Model coefficients are output of linear mixed model from gene signatures associated with the respective pathways. Black outline for each box represents $P<0.05$ for linear mixed model and $P<0.05$ for pathway enrichment (Method). (H) H\&E staining shows the thickening of BM and lamina propria labelled with asterisks, and the disappearance of the enterocyte brush border in the human inflamed colon. (I) Ki67 ${ }^{+}$proliferating cells and Lgr5 ${ }^{+}$ISCs were decreased, and Olfm $4^{+}$cells were increased in the inflamed colon. Lgr5 and Olfm4 were stained via in situ hybridization. (J) YAP showed more nuclear localization in the inflamed colon. $n=3$ for human colon resection samples. Scale bar, $200 \mu \mathrm{m}$.

We next assessed the expression of the YAP-up or down regulated genes (curated by Gregorieff et al. 
${ }^{19}$ ) in all the cell types. The YAP-downregulated genes (e.g., Fabp1 and Ces1f) were highly expressed in enterocytes and their progenitors, whereas the YAP-upregulated genes (e.g., Msln and Syt8) were enriched in goblet cells, IEGCs, and M cells (Fig. 4E and more YAP-regulated genes in Extended Data Figs. 19 and 20). These results further suggested that YAP could positively drive the differentiation of ISCs towards goblet cells as well as M- cells instead of enterocytes. Furthermore, the expression of the downstream genes of nuc-YAP, Id2, Birc5, and Areg ${ }^{20}$ significantly increased on the stiff matrix (Extended Data Fig. 21). These results together with our experimental observations (Fig. 2F) indicate that nuc-YAP drives the ISC differentiation towards goblet cells. in ISCs (Fig. 4F). Wnt is indispensable for maintaining the stemness of $\mathrm{Lgr}^{+} \mathrm{ISCs}^{21}$. Therefore, the stiffening-induced suppression of Wnt signaling could mediate the loss of Lgr $5^{\text {high }}$ ISCs. Furthermore, the suppression of the Notch pathway could drive the differentiation of ISCs towards goblet cells instead of enterocytes ${ }^{22}$, which is also in accordance with the stiffening-enhanced goblet cell differentiation. In addition, the differential expression analysis suggests a stiffening-induced metabolic reprogramming of ISCs, including an increase in glycolysis and a decrease in mitochondrial respiration (Fig. 4F). Furthermore, compared to the soft matrix, pathways involved in carbon metabolism are more enriched on the stiff matrix (Pathway enrichment analysis in Extended Data Fig. 22). The mechanotransduction signaling pathways including actin cytoskeleton, focal adhesion and tight junctions were also upregulated on the stiff matrix, which could potentially contribute to YAP activation (Extended Data Fig. 22). 
267 Smillie et al. ${ }^{3}$ ) and colon resection samples, from IBD patients and healthy individuals. The human single

cell profiles were clustered into ten epithelial cell subsets which were annotated using the known marker genes (tSNE in Extended Data Fig. 23A and marker genes in 23B). The relative proportions of each cell type demonstrate that UC is associated with a decrease in ISCs, an increase in goblet cells, and a trend towards a decrease in enterocytes (Fig. 4G and Extended Data Fig. 23C, with and without accounting for sample size, respectively). Pathway enrichment analysis demonstrates a strong activation of pathways involved in extracellular matrix biosynthesis (including collagen I and IV) in UC, indicating fibrosis and stiffening (Fig. 4G). Concomitantly, Wnt signaling was suppressed in the ISCs of UC patients (Fig. 4G and Extended Data Fig. 23D). The cluster of genes that have been previously shown to be directly upregulated by YAP ${ }^{19}$ were highly enriched in both ISCs and goblet cells of UC-derived tissues, indicating the upregulation of YAP in these cells (Fig. 4G). In addition, there is a strong activation of the mechanosignaling pathway, including integrins, YAP, and TEADs (the primary transcription factors for YAP), in both the ISCs and the goblet cells of UC, but not in enterocytes (Extended Data Fig. 23E). Together, these results suggest a mechanosignaling-induced ISC reprograming in UC, where the BM stiffening-induced upregulated integrin signaling promotes the expression of YAP as well as its interaction with TEAD in the nuclei, which potentially suppresses Wnt signaling in ISCs and drives their differentiation towards goblet cells.

Human UC colon samples showed the disappearance of the enterocyte brush border and the thickening of $\mathrm{BM}$ and lamina propria (Fig. $4 \mathrm{H})$. The excessive collagen deposition confirmed the occurrence of fibrosis (Extended Data Fig. 24A-C). Meanwhile, similar to our in vitro observations, Muc2 ${ }^{+}$goblet cells were overwhelmingly present in the inflamed colon compared to the healthy colon (Extended Data Fig. 
24D). The number of $\mathrm{Ki} 67^{+}$proliferating cells and $\mathrm{Lgr}^{+}$ISCs were significantly decreased in the inflamed samples (Fig. 4I). Nevertheless, Olfm4 expression was increased and extended into larger regions (Fig. 4I and Extended Data Fig. 24E). Notably, there is a strong YAP nuclear localization in the inflamed samples which could be induced by BM fibrosis and stiffening (Fig. 4J). In the extremely fibrotic strictured ileum of CD patient, the samples exhibited a complete loss of the normal invaginated crypt structures (Extended Data Fig. 25). Meanwhile, a large number of ectopic crypts were formed in the hyperplastic BM and lamina propria. These phenotypes of the strictured ileum closely resemble the in vitro stiffening-induced decrease of crypt size and the formation of ectopic crypts (Fig. 1G). The scRNAseq and histology data from human IBD patients, which strongly complement our in vitro observations, demonstrate that gut fibrosis is associated with a strong YAP nuclear translocation, loss of ISCs, extension of Olfm4, and enhanced differentiation towards goblet cells, all of which we have shown to be a direct consequence of tissue stiffening.

\section{Discussion}

We have developed a novel culture of quasi-3D gut organoids plated on top of soft hydrogel matrix.

The resulting in vitro cellular collective spontaneously reorganizes into a structure reminiscent of the crypt-villus anatomy and diverse cell types of native in vivo gut epithelium. While the 3D gut organoid cultured in Matrigel ${ }^{\circledR}$ has widely been used as an in vitro model of intestinal epithelium, it faces key limitations. Most notably, its intrinsic configuration does not mimic the in vivo anatomy and instead more closely resembles tumor conditions. Furthermore, the temporal and spatial nutrient and pressure gradients inside the Matrigel ${ }^{\circledR}$, could directly impact the growth of 3D organoids which underlie a substantial experimental variability ${ }^{23}$. By contrast, the novel system described here effectively circumvents these 
309 limitations and spontaneously generates crypt and villus structures with complex cellular composition similar to those in vivo.

Using this system, we observed that increasing matrix stiffness significantly reduced the TA progenitor cells and Lgr5 $5^{\text {high }}$ ISCs, while driving their differentiation preferentially towards goblet cells.

These results were confirmed in colitis mice and IBD patients. It is notable that it was previously reported that when the 3D gut organoids were embedded inside of the matrix, the matrix stiffening helped maintain the ISCs and suppressed their differentiation ${ }^{24}$. This discrepancy might be explained by the fact that the 3D organoids more resemble the tumor microenvironment. Additionally, our data show that the increased expression of nuc-YAP on the stiff matrix caused the reduction of ISCs, which is in-line with the results using Lats1/2 knockout mice ${ }^{25}$. We also elucidated, for the first time, the divergent roles of cyto-YAP and nuc-YAP in determining the direction of ISC differentiation. On the soft matrix, cyto-YAP dominates ISC differentiation into mature enterocytes, while on the stiff matrix, the nuc-YAP drives the differentiation towards goblet cells, giving rise to the new cell type, IEGC. Our data also have direct implications for the role of BM stiffening and YAP signaling in the progression of IBD to carcinoma. As suggested by our observations and the previous studies ${ }^{26,27}$, increasing YAP expression could decrease the incidence of $\mathrm{Lgr}^{+}$ISCs to form tumor. Nevertheless, the matrix stiffening expanded the expression of cyto-YAP from crypt-like regions into villus-like regions, which, in turn, expanded the expression of the oncogenes, Olfm4 and Egfr ${ }^{28-30}$. Moreover, the stiffening led to the formation of new crypts in the villus-like regions, which could contribute to the uncontrolled regeneration of gut epithelium and the development of carcinoma. 


\section{References}

3301 Bloemendaal, A. L., Buchs, N. C., George, B. D. \& Guy, R. J. Intestinal stem cells and intestinal homeostasis in health and in inflammation: a review. Surgery 159, 1237-1248 (2016).

2 Schmitt, M. et al. Paneth cells respond to inflammation and contribute to tissue regeneration by acquiring stem-like features through SCF/c-Kit signaling. Cell reports 24, 2312-2328. e2317 (2018).

3 Smillie, C. S. et al. Intra-and inter-cellular rewiring of the human colon during ulcerative colitis. Cell 178, 714-730. e722 (2019).

4 Scheibe, K. et al. Inhibiting interleukin 36 receptor signaling reduces fibrosis in mice with chronic intestinal inflammation. Gastroenterology 156, 1082-1097. e1011 (2019).

5 Stewart, D. C. et al. Quantitative assessment of intestinal stiffness and associations with fibrosis in human inflammatory bowel disease. PloS one 13 (2018).

6 Johnson, L. A. et al. Matrix stiffness corresponding to strictured bowel induces a fibrogenic response in human colonic fibroblasts. Inflammatory bowel diseases 19, 891-903 (2013).

7 Engler, A. J., Sen, S., Sweeney, H. L. \& Discher, D. E. Matrix elasticity directs stem cell Lineage specification. Cell 126, 677-689 (2006).

8 Mamidi, A. et al. Mechanosignalling via integrins directs fate decisions of pancreatic progenitors. Nature 564, 114-118 (2018).

9 Segel, M. et al. Niche stiffness underlies the ageing of central nervous system progenitor cells. Nature 573, 130-134 (2019).

10 Sato, T. et al. Paneth cells constitute the niche for Lgr5 stem cells in intestinal crypts. Nature 469, 415418 (2011).

11 Tetteh, P. W. et al. Replacement of lost Lgr5-positive stem cells through plasticity of their enterocytelineage daughters. Cell stem cell 18, 203-213 (2016).

12 Van Es, J. H. et al. Dll1+ secretory progenitor cells revert to stem cells upon crypt damage. Nature cell biology 14, 1099-1104 (2012).

13 Sato, T. et al. Single Lgr5 stem cells build crypt-villus structures in vitro without a mesenchymal niche. Nature 459, 262-265 (2009). 
14 Yilmaz, Ö. H. et al. mTORC1 in the Paneth cell niche couples intestinal stem-cell function to calorie intake. Nature 486, 490-495 (2012).

15 Dobrokhotov, O., Samsonov, M., Sokabe, M. \& Hirata, H. Mechanoregulation and pathology of YAP/TAZ via Hippo and non-Hippo mechanisms. Clinical and translational medicine 7, 1-14 (2018).

16 Scaltriti, M. \& Baselga, J. The epidermal growth factor receptor pathway: a model for targeted therapy. Clinical Cancer Research 12, 5268-5272 (2006).

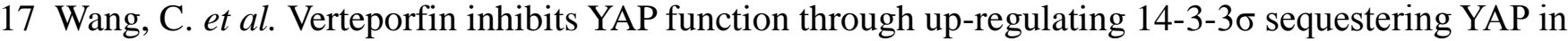
the cytoplasm. American journal of cancer research 6, 27 (2016).

18 Haber, A. L. et al. A single-cell survey of the small intestinal epithelium. Nature 551, 333-339 (2017).

19 Gregorieff, A., Liu, Y., Inanlou, M. R., Khomchuk, Y. \& Wrana, J. L. Yap-dependent reprogramming of Lgr5+ stem cells drives intestinal regeneration and cancer. Nature 526, 715-718 (2015).

20 Hong, W. \& Guan, K.-L. in Seminars in cell \& developmental biology. 785-793 (Elsevier).

21 Clevers, H., Loh, K. M. \& Nusse, R. An integral program for tissue renewal and regeneration: Wnt signaling and stem cell control. science 346, 1248012 (2014).

22 Yin, X. et al. Niche-independent high-purity cultures of Lgr5+ intestinal stem cells and their progeny. Nature methods 11, 106 (2014).

23 Shin, W. et al. Spatiotemporal gradient and instability of Wnt induce heterogeneous growth and differentiation of human intestinal organoids. Iscience 23, 101372 (2020).

24 Gjorevski, N. et al. Designer matrices for intestinal stem cell and organoid culture. Nature 539, 560564 (2016).

$25 \mathrm{Li}$, Q. et al. Lats $1 / 2$ Sustain Intestinal Stem Cells and Wnt Activation through TEAD-Dependent and Independent Transcription. Cell Stem Cell (2020).

26 Cheung, P. et al. Regenerative reprogramming of the intestinal stem cell state via Hippo signaling suppresses metastatic colorectal cancer. Cell Stem Cell 27, 590-604. e599 (2020).

27 Barry, E. R. et al. Restriction of intestinal stem cell expansion and the regenerative response by YAP. Nature 493, 106-110 (2013).

28 Kemper, K. et al. Monoclonal antibodies against Lgr5 identify human colorectal cancer stem cells. 
Stem cells 30, 2378-2386 (2012).

29 Van der Flier, L. G., Haegebarth, A., Stange, D. E., Van de Wetering, M. \& Clevers, H. OLFM4 is a robust marker for stem cells in human intestine and marks a subset of colorectal cancer cells. Gastroenterology 137, 15-17 (2009).

30 Karapetis, C. S. et al. K-ras mutations and benefit from cetuximab in advanced colorectal cancer. New England Journal of Medicine 359, 1757-1765 (2008).

31 Barker, N. et al. Identification of stem cells in small intestine and colon by marker gene Lgr5. Nature 449, 1003-1007 (2007).

$32 \mathrm{He}$, S. et al. The tumor suppressor p53 can promote collective cellular migration. PloS one 14, e0202065 (2019).

33 Schlegelmilch, K. et al. Yap1 acts downstream of $\alpha$-catenin to control epidermal proliferation. Cell 144, 782-795 (2011).

Acknowledgements This work was supported by funding from National Institutes of Health (R01DK123219, R01EY028234, and K01DK103947 to N.S., and R01HL148152 and U01CA202123 to J.J.F.), ECOR/MGH (2019A002949 to N.S.), and Polsky family fund (to N.S.). We thank Dr. Ramnik J. Xavier, the Director of the Center for the Study of Inflammatory Bowel Disease at Massachusetts General Hospital (National Institutes of Health, P30DK043351) for his constructive comments, providing human samples and sharing the human scRNAseq data. We thank Maris A. Handley and Jacqueline Choi from the HSCI-CRM Flow Cytometry Core and iHisto Inc for their supports.

Author Contributions S.H. and N.S. conceptualized the work and designed the experiments. S.H. and P.L. performed the experiments with inputs from J.W., P.C., J.O.R. and F.D.C. on generating transgenic mice, C.Y.P. and J.J.F. for live cell imaging, M.M. and Ö.H.Y. for flow cytometry, E.S. and D.T.B for training, and S.I. for in situ hybridization. S.H., H.W. and V.M. performed the DSS mouse model with inputs from Z.Z. for measuring thickness, and R.W. and Y.Z. for measuring stiffness. W.K., K.S., T.X. and P.B. performed the scRNAseq analysis for human and organoids with guidance from S.H., R.S. and N.S.. S.H. and N.S. wrote the manuscript. J.J.F., F.D.C., P.C., M.M.,C.Y.P., D.T.B., T.X., M.H. and I.A. commented on the manuscript.

The authors declare no competing financial interests. N.S. and S.H. are inventors on a patent application filed based on this investigation.

Correspondence and requests for materials should be addressed to N.S. (nsaeidi@mgh.harvard.edu). Supplementary Information is available for this paper. 


\section{Methods}

419 Mice including the strains of wild type C57BL/6J, Lgr5-EGFP-IRES-CreER ${ }^{31}$, YAP conditional knockout and YAP conditional overexpression were used for harvesting crypts. Polyacrylamide hydrogel matrix was fabricated as described before ${ }^{32}$. The crypts collected from the mouse small intestines were counted and seeded on the hydrogel matrix. After 11 days culture the cells were fixed for immunofluorescent staining, or the cells were trpsinized for flow cytometry, the transferring to 3D organoid culture in Matrigel, and single cell RNA sequencing. The fluorescent signals after staining were quantified using a customized MATLAB code. Initial processing of scRNA-Seq sequencing data was performed using CellRanger (v4.0.0). Further analysis was performed using the Seurat and Phenograph. C57BL/6J mice were given 3 cycles of DSS to induce chronic colitis. The mouse colon tissues were collected for immunohistochemistry and the tissue stiffness was measured using an Instron uniaxial tester. The human samples were provided by the Prospective Registry in IBD Study at MGH (PRISM).

\section{Detailed Methods}

\section{Mice}

432 10-14 weeks old mice including the strains of wild type C57BL/6J, Lgr5-EGFP-IRES-CreER ${ }^{31}$, YAP conditional knockout and YAP conditional overexpression were used for harvesting crypts. To generate the YAP conditional knockout mice, CAG-rtTA3 (Jackson Laboratories) mice were mated with (TetO)7-

435 Cre (Jackson Laboratory) and Yap ${ }^{\mathrm{fl} / \mathrm{fl}}$ mice ${ }^{33}$. To generate the YAP conditional overexpression mice, the tetO-YAP-GFP mice (Jackson Laboratory, 031279) expressing mutant S112A YAP crossbred with VillinrtTA*M2 mice (Jackson Laboratory, 031285). $1 \mu \mathrm{g} / \mathrm{ml}$ DOX was added to induce YAP knockout or overexpression in the organoid culture. 


\section{Preparation of the hydrogel matrix}

440 Polyacrylamide (PA) hydrogel matrix were fabricated on a $35 \mathrm{~mm}$ dishes with glass bottom (Cellvis, D35-

20-1.5-N) as described ${ }^{32}$. Briefly, the recipe for different Young's modulus were $3 \%$ acrylamide (Bio-Rad, 1610140 ) and $0.058 \%$ bisacrylamide (Bio-Rad, 1610142) for $0.6 \mathrm{kPa}, 7.5 \%$ acrylamide and $0.034 \%$ bisacrylamide for $2.4 \mathrm{kPa}$, and $7.5 \%$ acrylamide and $0.148 \%$ bisacrylamide for $9.6 \mathrm{kPa} .0 .1 \%$ ammonia persulfate (sigma, 09913) and 0.05\% TEMED (Bio-Rad, 1610800) were added to start the polymerization process. Then, $70 \mu \mathrm{l}$ gel solution was added into the dishes, and a cover slip of $18 \mathrm{~mm}$ in diameter was placed on the gel solution to flatten the surface and resulted in a gel of $300 \mu \mathrm{m}$ in thickness (VWR, 48380 046). The polymerization required 40 minutes to 1 hour. Then, sulfo-SANPAH (Proteochem, C1111) was used to activate the gel surface under a 15 W 365 nm UV (VWR, 95-0042-07/36575-050) for 10 minutes. After the activation, $200 \mu 10.1 \mathrm{mg} / \mathrm{ml}$ type I collagen (Advanced biomatrix, 5022) was added onto the gel overnight to covalently attach to the gel surface for cell culture.

\section{Harvest of crypts}

The proximal 12 15 cm small intestines were collected from mice. The intestinal lumen was opened

longitudinally. The mucous was removed using the back of blades. Then, the intestine was washed with

454 ice cold PBS without calcium and magnesium (Corning, 21-040), and cut into $5 \mathrm{~mm} \sim 1 \mathrm{~cm}$ fragments and placed into $50 \mathrm{ml}$ conical tubes that were filled with ice-cold $50 \mathrm{ml}$ of PBS/EDTA (10 mM, Thermo fisher, 15575020). The fragments were incubated and shaken on ice for 40 minutes, and washed with icecold $50 \mathrm{ml}$ of PBS. Then, the fragments were vigorously shaken in $25 \mathrm{ml}$ PBS and filtered twice through a $70 \mu \mathrm{m}$ mesh (BD Falcon) into a $50 \mathrm{ml}$ conical tube to remove the villi and tissue pieces. The crypts were 
was then ready for seeding on the hydrogel.

\section{Culture of Crypts}

The crypt pellets were suspended in the seeding media and counted using cytometer to control the crypt density as $10,000 / \mathrm{ml}$. $150 \mu \mathrm{l}$ to $200 \mu \mathrm{l}$ crypt suspension was added on to the PA gel in the $35 \mathrm{~mm}$ dishes. After 4 hours, the floating cells/clusters were washed with PBS (Corning, 21-040-cv). $1.5 \mathrm{ml}$ ENR media/dish was added and changed every other day. To make the ENR media, advanced DMEM/F12 (Gibco, 12634-028) was supplemented with 50 ng/ml EGF (Peprotech, 315-09), 100 ng/ml Noggin (Peprotech, 250-38), 10\% R-spondin conditional media (iLab in Harvard digestive center), 1\% Glutamax (Gibco, 35050-061), 1\% HEPES (Gibco, 15630-080), 0.2\% Primocin (Invivogen, ant-pm-2), 0.2\% Normocin (Invivogen, ant-nr-2), 1\% B27 (Gibco, 12587010), 0.5\% N2 (Gibco, 17502-048), and 1.25 mM N-Acetyl-Cystein (Sigma, A8199). To make the seeding media, the ENR media was supplemented with 3 $\mu \mathrm{M}$ Chir-99021 (Selleckchem, S1263) and $10 \mu \mathrm{M}$ Y-27632 (Sigma, Y0503). We captured the phase images and the GFP images for Lgr5-EGFP fluorescent signal every 20 mins for days using 20X objective of Leica microscope (Leica DMI8) with a live cell imaging system. Depending on different purposes, 1 $\mu \mathrm{g} / \mathrm{ml} \mathrm{DOX}$ and $1 \mu \mathrm{M}$ VP was, respectively, added to induce YAP KO or OE, or inhibit YAP nuclear translocation during the culture. After 10-11 days culture, cells were fixed for immunofluorescent staining, or collected using TrypLE Express (Invitrogen, 12-605-010) for different purposes including flow cytometry, the transferring to 3D organoid culture in Matrigel, and single cell RNA sequencing.

\section{Immunofluorescence (IF), immunohistochemistry (IHC) and In situ hybridization}

\section{(ISH)}

For in vitro IF, cells were fixed in $4 \%$ paraformaldehyde/PBS for 10 minutes and cold $70 \%$ ethanol for 30 
minutes, blocked in 2\% BSA for 30 minutes and permeabilized in $0.3 \%$ Triton X-100/PBS for 20 minutes.

482

The cell layers were stained with primary antibody, then stained with secondary antibodies. Laser scanning microscopy images were captured by using the inverted confocal microscope (Nikon C2, 20X or 60X objective). The average intensity of the fluorescent signals in these images were then quantified using a customized MATLAB code which can identify the crypt-like regions based on the nuclei density (Extended Data Fig. 26). All the fluorescent images represented at least nine field views from three different animal (3 filed views/animal). Student's $t$-test was used to determine statistical significance with a cut-off of $P<0.05$. In vivo IHC and IF was performed by iHisto Inc. Samples were processed, embedded in paraffin, and sectioned at $4 \mu \mathrm{m}$. Paraffin sections were then deparaffinized and hydrated. Antigen retrieval was achieved by boiling the sections in $10 \mathrm{mM}$ sodium citrate. Sections were then washed with PBS three times, treated with $3 \% \mathrm{H}_{2} \mathrm{O}_{2}$ for $15 \mathrm{~min}$ and $5 \%$ bovine serum albumin for 20 minutes. The sections were incubated with primary antibodies overnight at $4{ }^{\circ} \mathrm{C}$. Subsequently, the sections were immunohistochemically stained with secondary antibodies for $50 \mathrm{~min}$ at room temperature. Rabbit primary antibodies were used for staining Villin (ab130751), Muc2 (ab76774, ab272692), ChromograninA (ab15160), Lysozyme (ab2408), non-phosphorylated YAP (ab205270), Trefoil Factor 3 (ab272927) and Egfr (ab40815), Ki-67 (cs9129), Olfm4 (ab105861, cs14369), Ser127 Phospho-YAP (cs13008), collagen I (ab34710) and collagen IV (ab6586). Mouse primary antibodies were used for total YAP (ab56701) and Villin (Proteintech, 66096-1-Ig). Goat anti rabbit/mouse Alexa Fluor 405, 488, 594 and 647 (Thermo fisher) were used as secondary antibodies. DAPI (Fisher scientific, D1306) and UEA-1 Fluorescein (Vector labs, FL-1061-5) were respectively used for staining nuclei and Paneth cells. Single-molecule in situ hybridization was performed using Advanced Cell Diagnostics (ACD) RNAscope 2.0 HD Detection Kit 
502 (Fast Red dye) for the probes of Lgr5 (ACD, 311021) and Olfm4 (ACD, 311041).

\section{Flow cytometry}

504 The cells on the PA hydrogel were trypsinized using TrypLE for 10 minutes at $37^{\circ} \mathrm{C}$. After collecting the

505

506

507

508

509

510

511

512

513

514

515

516

517

518

519

520

521

522

cells, cold SMEM (1:5) was added to stop the trypsinization, and followed by centrifuging for 5 min at $300 \mathrm{~g}$. The cell pellets were re-suspended and stained for $15 \mathrm{~min}$ on ice in $1 \mathrm{ml}$ antibody cocktail. To make the antibody cocktail, SMEM (Sigma, M8167) was supplemented by CD45-PE (1:500, eBioscience, 30-

F11), CD31-PE (1:500, Biolegend, Mec13.3), Ter119-PE (1:500, Biolegend, Ter119), CD24-Pacific Blue (1:300, Biolegend, M1/69), EPCAM-APC (1:300, eBioscience, G8.8) and CD117(C-kit)-APC-CY7 (1:300, Thermo fisher, 47-1171-80) . After the staining 10ml SMEM were added and the samples were centrifuged for $5 \mathrm{~min}$ at $300 \mathrm{~g}$. The pellets were resuspended with $1 \mathrm{ml}$ SMEM supplemented by 7-AAD

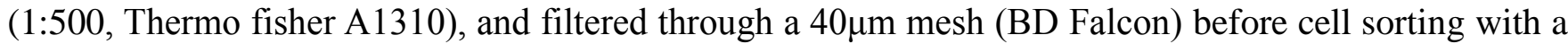

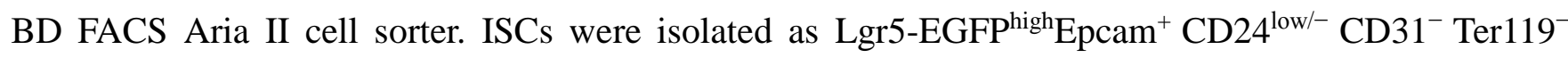
$\mathrm{CD}_{4}{ }^{-}$and $7-\mathrm{AAD}^{-}$, TA progenitors were isolated as $\mathrm{Lgr5}_{-\mathrm{EGFP}}{ }^{\text {low }}$ Epcam $^{+} \mathrm{CD} 24^{\text {low/- }} \mathrm{CD}^{-} 1^{-}$Ter119 $^{-}$ $\mathrm{CD}^{-} 5^{-}$and 7-AAD ${ }^{-}$, Paneth cells were isolated as CD24 ${ }^{\text {high }}$ Sidescatter $^{\text {high }}$ C-kit ${ }^{+}$Lgr5-EGFP $^{-}$Epcam $^{+}$ $\mathrm{CD}^{-} 1^{-} \mathrm{Ter}_{119}{ }^{-} \mathrm{CD}^{-} 5^{-}$and $7-\mathrm{AAD}^{-}$.

\section{Culture of 3D organoids in Matrigel transferred from the 2D hydrogel}

The cells on the hydrogel martix were trypsinized using TrypLE for 5 minutes at $37^{\circ} \mathrm{C}$. The wells were sealed with lid, and the bottom of the wells were vigorously hit onto the table to detach the cells. After collecting the cells/clusters cold SMEM (1:5) was added to stop the trypsinization, and followed by centrifuging for $5 \mathrm{~min}$ at $300 \mathrm{~g}$. The cell pellets were resuspended in ENR media and Matrigel (1:1, Corning 356231), then seeded $25 \mu \mathrm{l} /$ well in a 48 -well plates and put at $37^{\circ} \mathrm{C}$ for solidification. After 20 
minutes, $150 \mu \mathrm{l} /$ well ENR media was added, and changed every 3 days. The 3D organoids were imaged

524 on day 5.

\section{Single cell RNA sequencing for the gut organoids}

526 The scRNA-seq library construction was performed on the Chromium 10x instrument using Chromium 527 single cell 3' reagent v3.0 kits, followed by sequencing on Illumina HiSeq 2500 instruments, which resulted in approximately 160 million reads per sample. Initial processing of scRNA-Seq sequencing data was performed using CellRanger (v4.0.0)

(https://support.10xgenomics.com/single-cell-gene-expression/software/overview/welcome). In brief,

reads were aligned to mm10 mouse reference genome with the mapping rate of $\sim 70 \%$, followed by the generation of read counts per gene in each cell. Further analysis was performed using the Seurat 3.2.3 package (https://satijalab.org/seurat/). We filtered out cells with <200 expressed genes and genes expressed in $<3$ cells, followed by the exclusion of cells with high content of mitochondrial transcripts (>

$20 \%$ of total reads). Counts across all cells for each sample were normalized using NormalizeData function and the effect of the cell cycle was removed by regressing the difference out between the $S$ phase and G2M phase from normalized data. Using the FindVariableFeatures function we selected 2000 features to be used in a Principal Component Analysis (PCA). UMAP dimensionality reduction and cell clustering were performed using RunUMAP and FindClusters functions, respectively. VlnPlot and FeaturePlot functions were used to generate violin plots and feature plots for the datasets. Heatmaps of gene expression were generated using DoHeatmap function. Cells from all samples were then integrated using Seurat 
function. Biological annotation of cell clusters was based on the expression of known cell type markers.

For the differential expression analysis, FindMarkers function was applied to the integrated samples in order to identify differentially expressed genes between the cell subsets.

\section{Single cell RNA sequencing for human colon epithelium}

\section{Cell clustering and differential expression analysis}

We re-analyzed scRNAseq data of colon biopsy specimen generated by Smilie et al $^{3}$ (raw data from https://portals.broadinstitute.org/single_cell/study/SCP259). From the dataset, we used epithelial samples containing healthy tissue and inflamed tissue samples from patients with ulcerative colitis. We followed the same procedure to identify cell clusters as outlined in Smilie et al using Seurat ( https://satijalab.org/seurat/, v.3.2.3) and Phenograph. The only exception being a larger k=1000 was used when applying Phenograph to $\mathrm{KNN}$-graphs, and then re-clustering with $\mathrm{k}=50$ is used to identify rare epithelial cell types. Cell clusters were identified by gene expression with known cell type markers. Barnes-Hut t-Distributed Neighbor Embedding on PCS (perplexity=10, iterations=5000) provided visualization of data embedding. The coarser $\mathrm{k}$ resulted in larger cell clusters where immature forms of cell types are no longer differentiated from the terminally differentiated cell types. The MAST model is fit to identify cell type markers and DE genes in inflamed tissue samples with control tissue, discrete coefficient of MAST model output is reported in text and figures unless otherwise stated.

\section{Identifying statistically significant differences in cell proportions}

As samples with exceedingly small number of cells show few cell types and disproportionate cell type proportions, we excluded samples containing less than 250 cells from subsequent analysis. Then changes in cell proportion between healthy and inflamed tissue are assessed by two methods. We first used montecarlo test, where $\mathrm{H} 0$ is differences in proportions for each cell type between inflamed and healthy condition is a consequence of random sampling. We combined cells from both conditions together, and then randomly segregated cells back into the two conditions while maintaining original sample sizes and repeated the process 1000 times. We recalculated the proportion difference between the two conditions and compare to observed proportional difference for each cell type, and the $P$-value reflect number of 
simulations where simulated proportional difference was more than observed. This test reflects how enriched each cell type is within each condition (healthy or inflamed), but does not account for the specimen from which each cell is isolated. In the second method, we calculated the relative variation in each cell type proportion between all pairs of healthy donors as a control. Then, we calculated the relative variation in each cell type by dividing the fraction of the cell type in each inflamed specimen by that of a healthy specimen. After $\log _{2}$ transformation, we conducted a two-sided Kolmogorov-Smirnov (KS) test of the relative variation in composition between the control (healthy) and inflamed groups (ks.test function).

\section{Identifying significant changes in pathway gene signatures}

The ECM pathway (YAP1ECM_AXIS) was obtained from WikiPathways and WNT signaling pathway is obtained from KEGG. The gene signature of top 200 YAP-upregulated genes were curated by Gregorieff et al ${ }^{19}$. Pathway enrichment analysis (PEA) were performed using gene sets from these pathways with the fgsea package in $\mathrm{R}$, and the shared genes between significant cell types are used as the gene signatures for the pathways of interest. Expression of each gene was then scaled by its root mean squared expression across all cells, and mean scaled expression for all signature genes in the pathway is calculated to give a signature score for each cell. Then, we used mixed linear models to identify changes in expression levels of gene signatures in the inflamed state. In the model, the fixed effect term is used to represent the condition of each cell (healthy or inflamed) and the random intercept that varies with each sample is used to account for the sample each cell was isolated from. ANOVA is used to estimate the fixed term $P$-value.

\section{Chronic Colitis mouse model induced by DSS and samples from IBD patients}

To induce chronic colitis, Wild type 8-weeks old C57BL/6J mice were given 3 cycles of DSS. Each cycle included 2.5\% DSS in drinking water for 7 days and distilled water for the following 14 days. After the third cycle of giving DSS verteporfin (VP, $25 \mathrm{mg} / \mathrm{kg} / \mathrm{d}$ in DMSO; Sigma-Aldrich) or vehicle as control was administered via i.p. injection for 14 days. Then colon tissues were collected for measuring stiffness and immunohistochemistry (IHC). Antibodies were used same as in IF. ID of human patients for the healthy, inflamed, and strictured gut samples are, respectively, 109922, 117324, and 110201. Protocol No. for involving human subjects is 2004P001067 reviewed by IRB. 


\section{Uniaxial tensile testing for colon stiffness measurement}

598 The colons collected from the chronic colitis experiments were cut open along the longitudinal direction 599 into flat rectangular patches using a surgical scalpel. Sandpaper tabs were glued to both ends of a sample 600 to prevent slipping during testing. The effective length $\left(l_{0}\right.$, i.e., sandpaper-to-sandpaper distance) and 601 width $(w)$ of each sample were measured using a caliper. The section view of the colons supported with a 602 10ul tips was imaged, and the thickness $(t)$ of the section was measured using Image-J. Samples were 603 mounted on an Instron uniaxial tester (Instron, Norwood, MA) by clamping the sandpaper tabs with the 604 605 606 away the other end with an extension rate of $0.02 \mathrm{~mm} / \mathrm{s}$. The pulling force $(F)$ and the displacement $(d)$ 607 of the moving end of the sample were recorded at a frequency of $5 \mathrm{~Hz}$. The end of the regime of elastic 608 deformation was marked by a drop in the slope of the force-displacement curve. The stain $(\varepsilon)$ of the sample 609 was obtained as

$$
\varepsilon=\frac{d}{l_{0}}
$$

611 Assuming tissue incompressibility, the Cauchy stress $(\sigma)$ can be calculated as

$$
\sigma=\frac{F}{w t}(1+\varepsilon)
$$




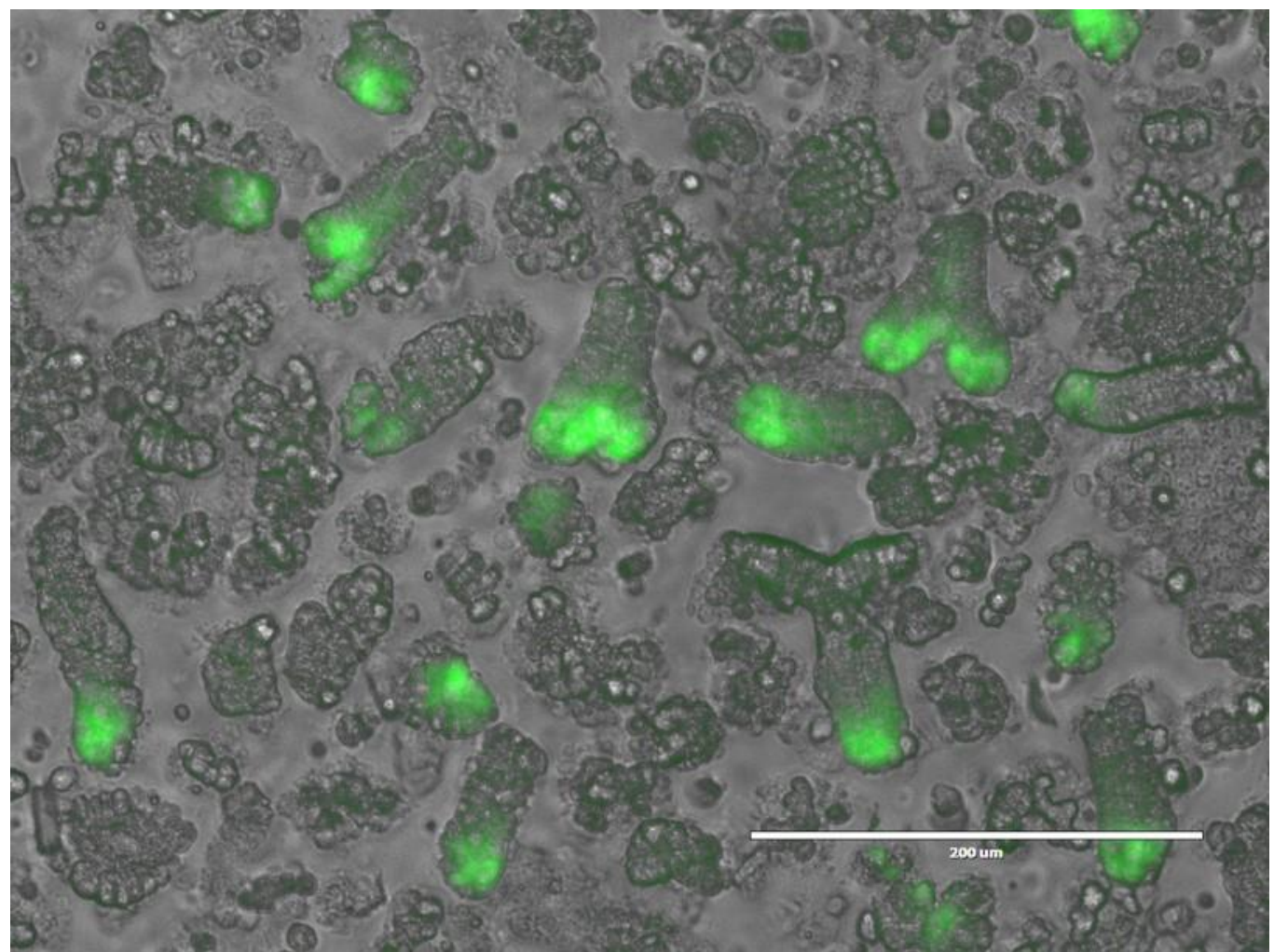

617 Extended Data Figure 1. The crypts with Lgr5-EGFP ${ }^{+}$ISCs were harvested from $L g r 5-E G F P I R E S-^{-}$ 618 creERT2 mice, and seeded on the hydrogel matrix. 
A

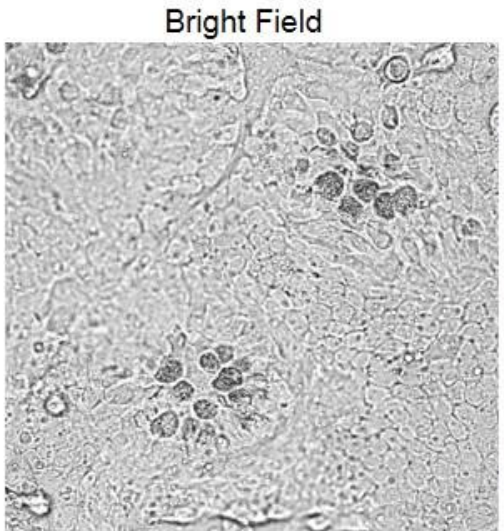

UEA

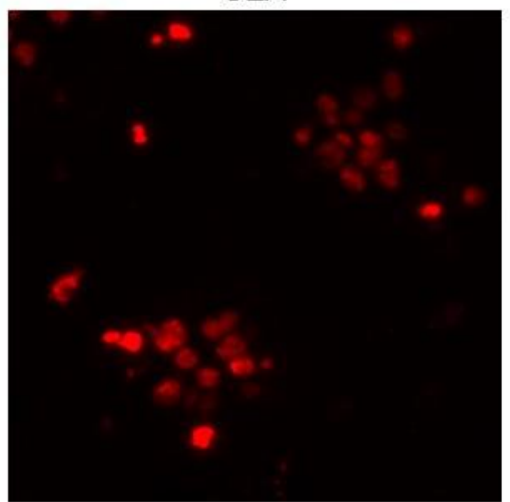

B

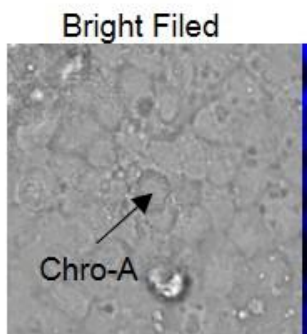

\section{Bright Filed}

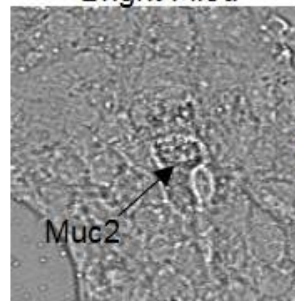

DAPI
Lgr5-Egfp

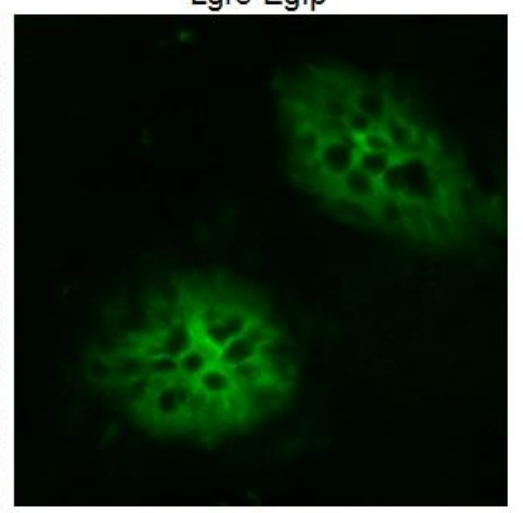

Ki67

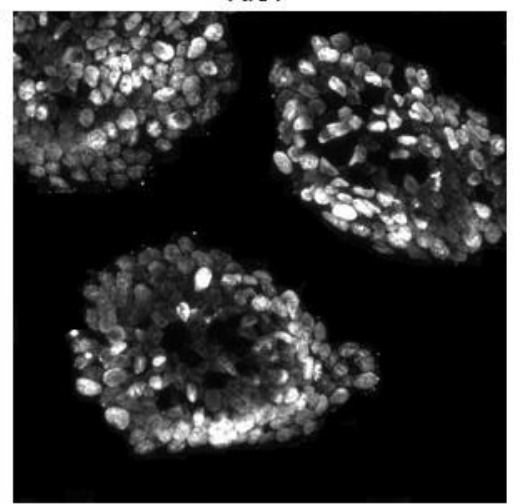

Chro-A

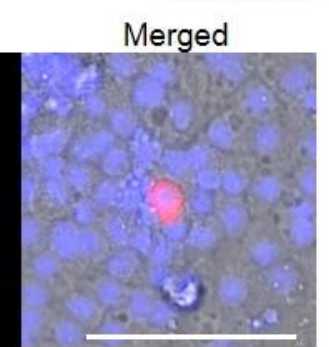

Muc2

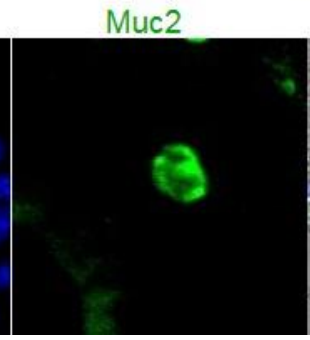

Villin

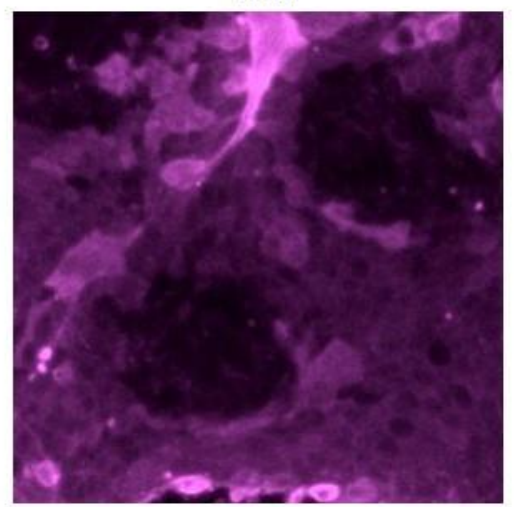

Lgr5+Ki67+UEA+Villin

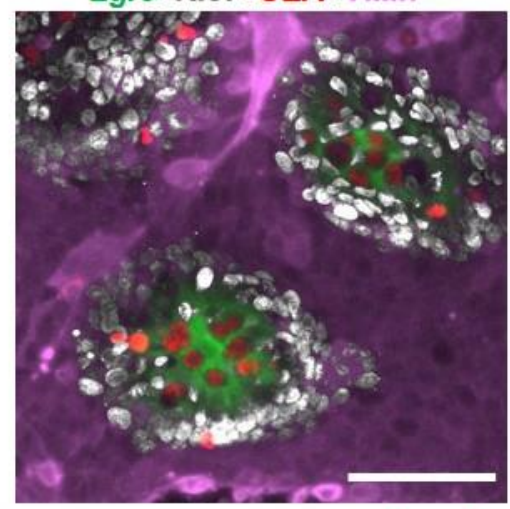

DAPI+Chro-A

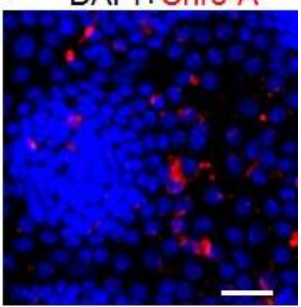

DAPI+Muc2

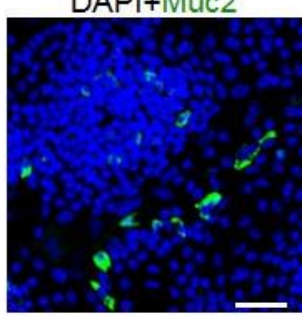

Extended Data Figure 2. (A) Lgr5-EGFP ${ }^{+}$ISCs were intermixed with the optically dark $\mathrm{UEA}^{+}$Paneth cells, which were surrounded by Ki67+ TA cells in the crypt-like regions. Lgr5-EGFPhigh ISCs weakly expressed Ki67. The villus-like regions were populated by Villin ${ }^{+}$enterocytes. (B) Chro- $\mathrm{A}^{+}$EEC and 

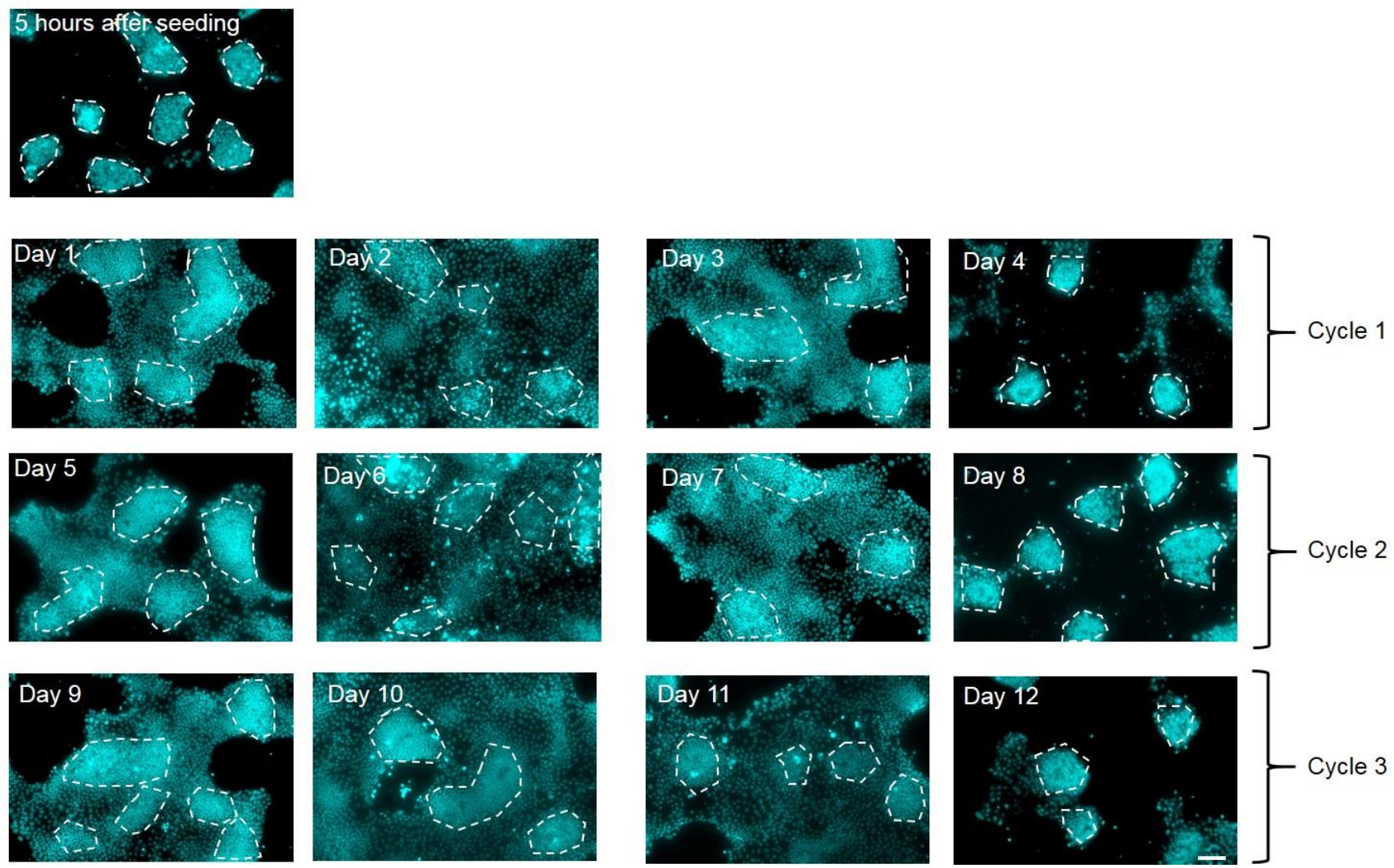

Extended Data Figure 3. The villus-like regions exhibited a turnover rate of approximately 3 days

$631(n=5)$. The white dashed lines trace the crypt-like regions. Scale bar, $100 \mu \mathrm{m}$. 

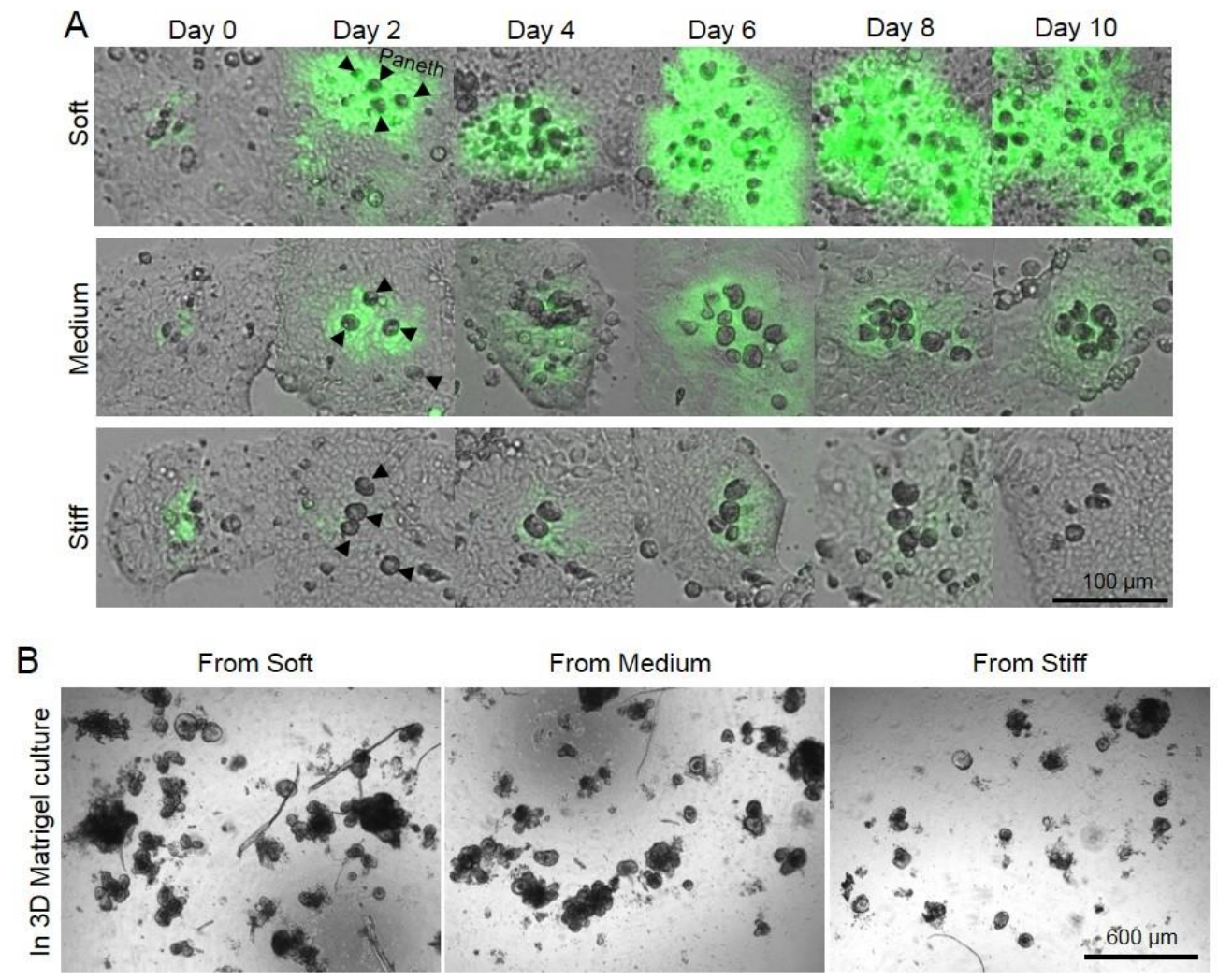

-Soft - Medium —Stiff

Budding No. / organoid

Ectopic crypt No. I Villus region

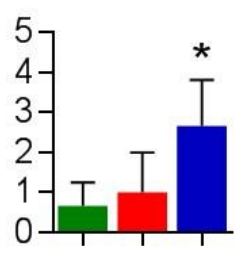

Extended Data Figure 4. (A) Long-term live-cell imaging exhibited completely different phenotype of Lgr5-EGFP ${ }^{+}$ISCs between the soft matrix and the stiff matrix. More specifically, on the soft matrix, the Lgr5-EGFP ${ }^{+}$ISCs continuously increased and expanded throughout the culture period. In contrast, Lgr5$\mathrm{EGFP}^{+}$ISCs on the stiff matrix progressively diminished over time, nearly disappearing by the $10^{\text {th }}$ day. The medium matrix was able to maintain some Lgr5-EGFP ${ }^{+}$ISCs. (B) The 3D organoids from the soft or medium matrix budded, but those from the stiff matrix grew as cysts with less budding $(n=3)$. (C) New crypt generation in the villus-like regions appeared more on the stiff matrix than on the soft matrix (Movies. S2 and S3, $n=3)$. * V.S. Soft and \# V.S. Medium, $P<0.05$. 

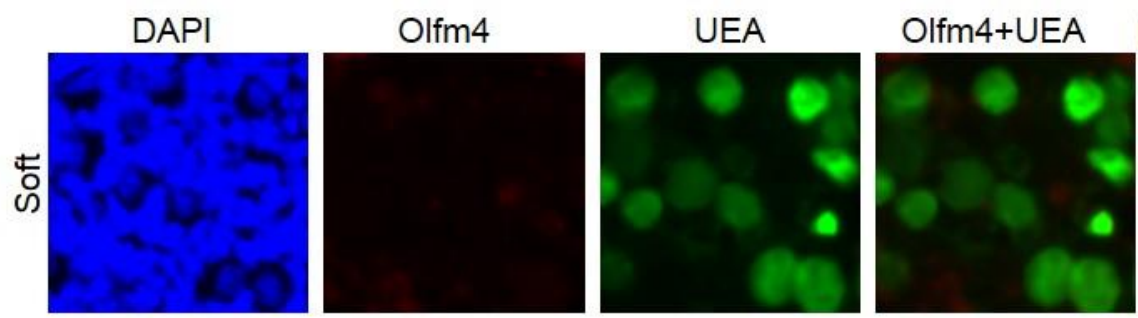

DAPI+Olfm4+UEA

644 Extended Data Figure 5. In the interior of the crypt-like regions, $\mathrm{Olfm} 4^{+}$cells became adjacent with $645 \mathrm{UEA}^{+}$Paneth cells on the stiff matrix $(n=3)$. Scale bar, $50 \mu \mathrm{m}$ 

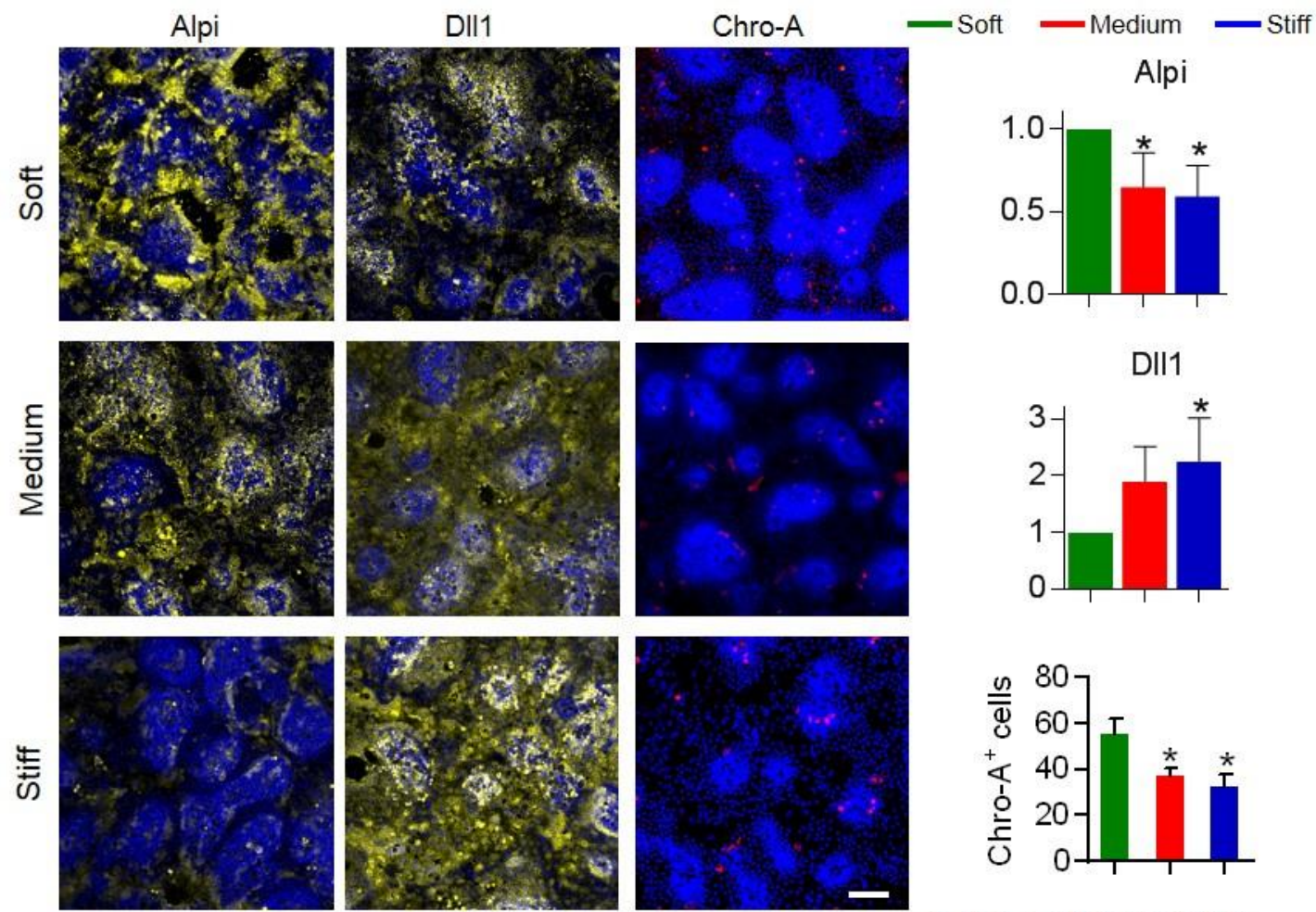

646

* V.S. Soft for $p<0.05$

647 Extended Data Figure 6. The stiffening decreased Alpi and Chro-A, and increased Dll1 ( $n=3)$. Scale 648 bar, $100 \mu \mathrm{m}$.

649

650

651

652 

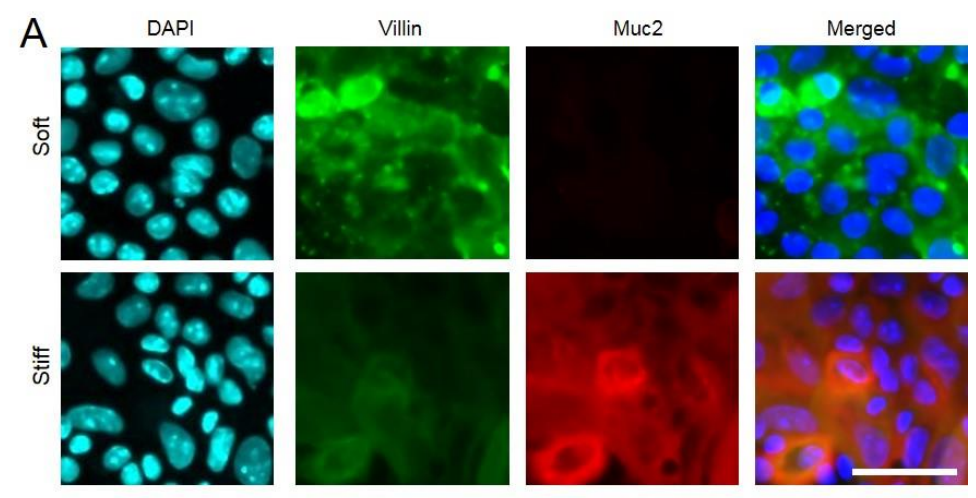

B
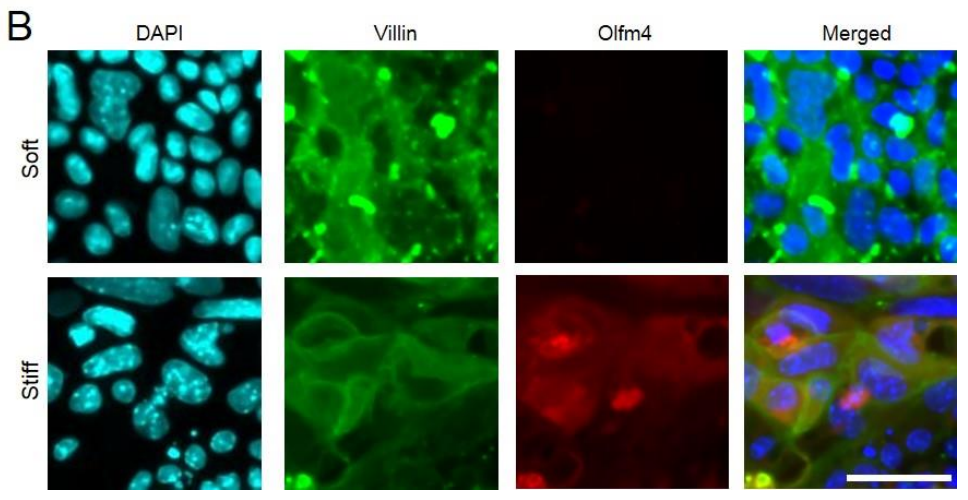

655 Extended Data Figure 7. Counterstaining in the villus-like regions for Villin and Muc2 (A), and Villin 656 and Olfm4 (B). $n=3$. Scale bar, $50 \mu \mathrm{m}$.

657

658 

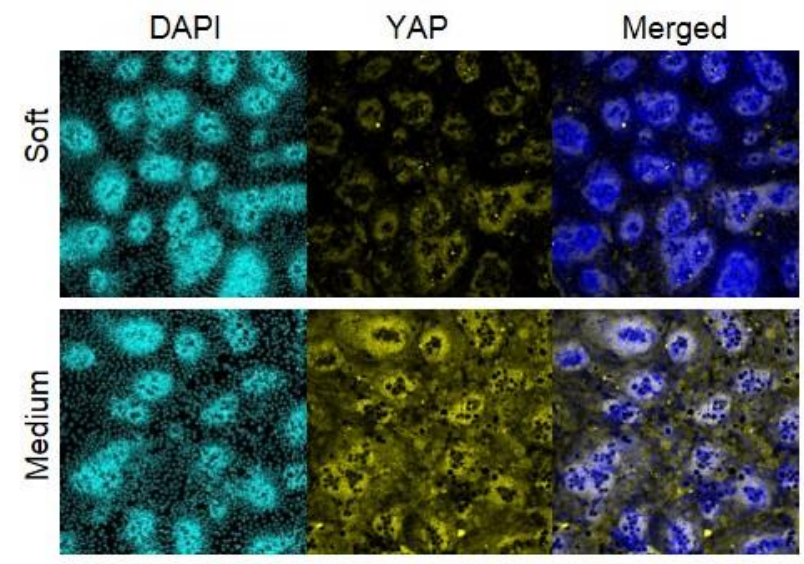

660 Extended Data Figure 8. Stiffening increased YAP expression and promoted YAP nuclear translocation 661 on the stiff matrix $(n=5)$. Scale bar, $100 \mu \mathrm{m}$. 


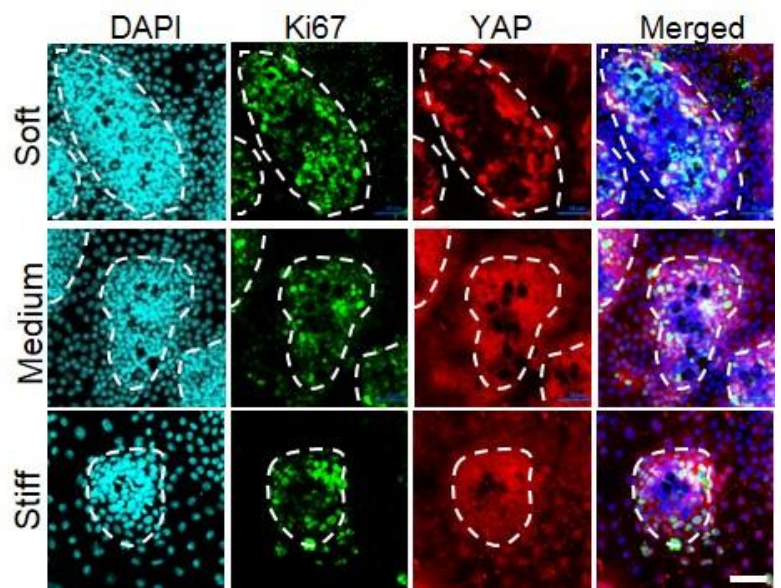

664 Extended Data Figure 9. Ki67 was positively correlated with cyto-YAP $(n=3)$. The white dashed lines 665 trace the crypt-like regions. Scale bar, $25 \mu \mathrm{m}$.

666

667

668 


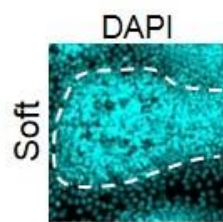

\section{Olfm4}
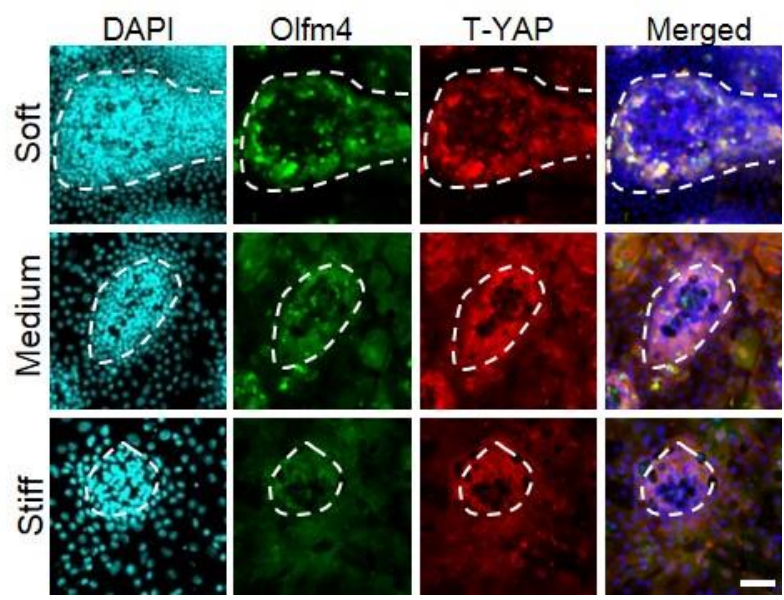

671 Extended Data Figure 10. Olfm4 was positively correlated with cyto-YAP $(n=3)$. The white dashed lines 672 trace the crypt-like regions. Scale bar, $25 \mu \mathrm{m}$.

673

674

675

676

677

678 

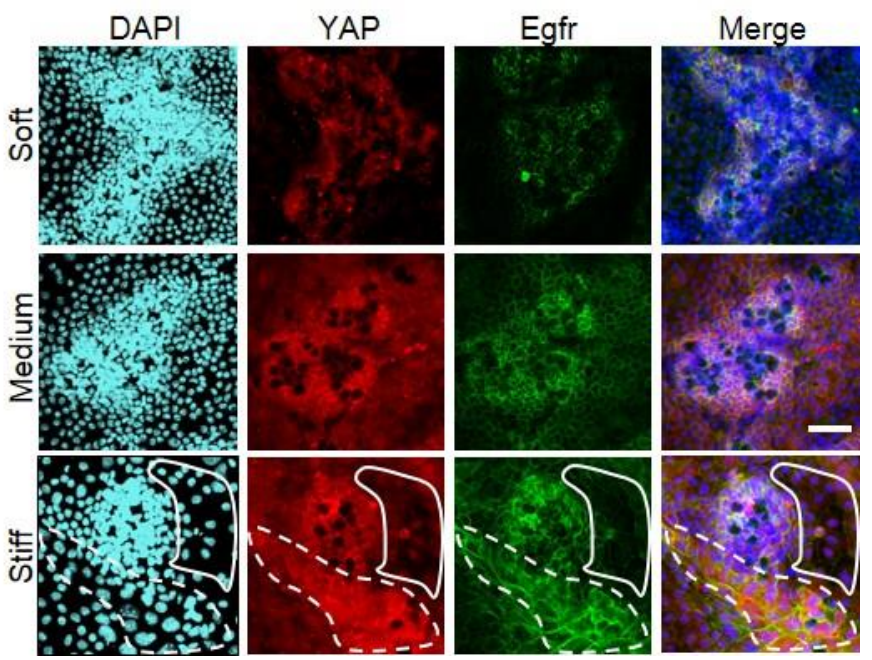

680 Extended Data Figure 11. Egfr was positively correlated with cyto-YAP $(n=3)$. On the stiff matrix, the 681 white dashed line traces the region with high expression of cyto-YAP, and the solid line traces the region 682 with YAP nuclear localization. Scale bar, $25 \mu \mathrm{m}$. 

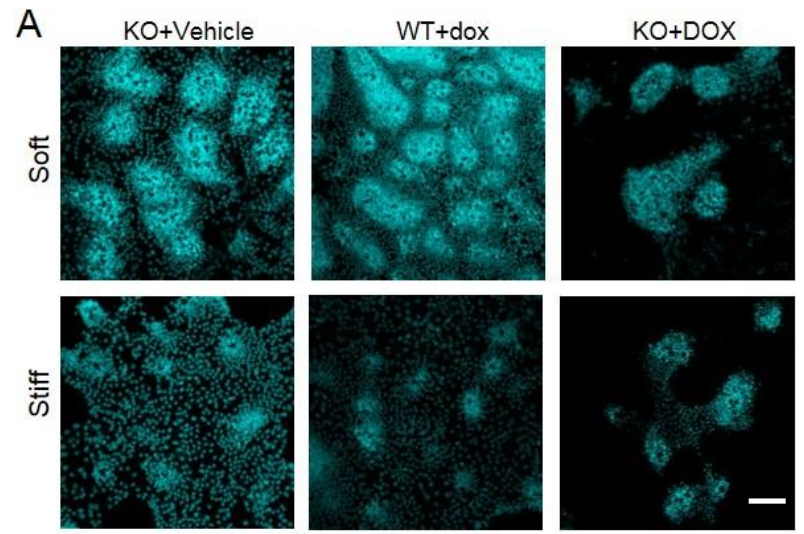

B
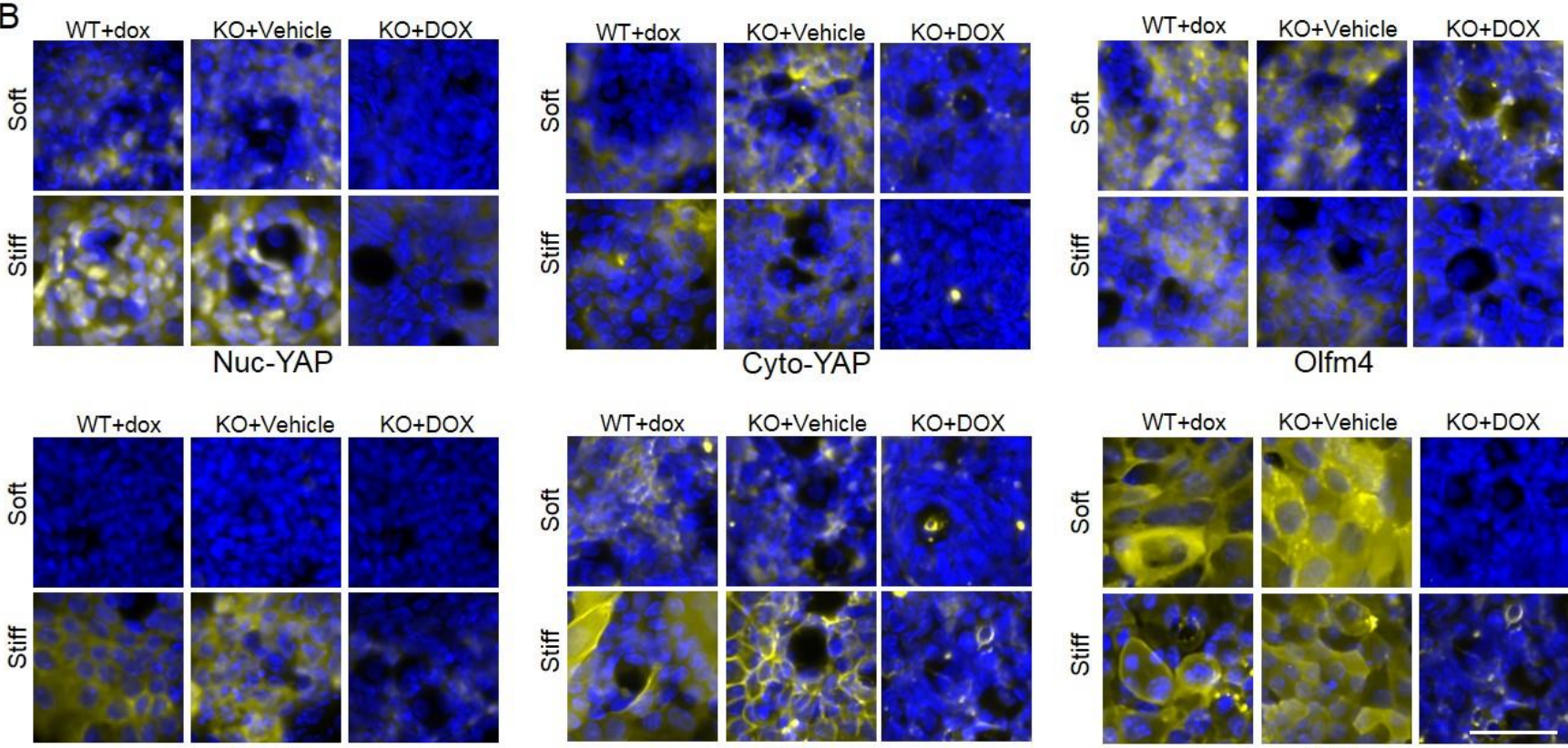

Muc2
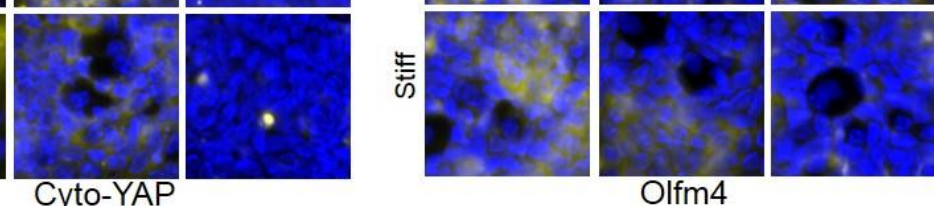

Olfm4
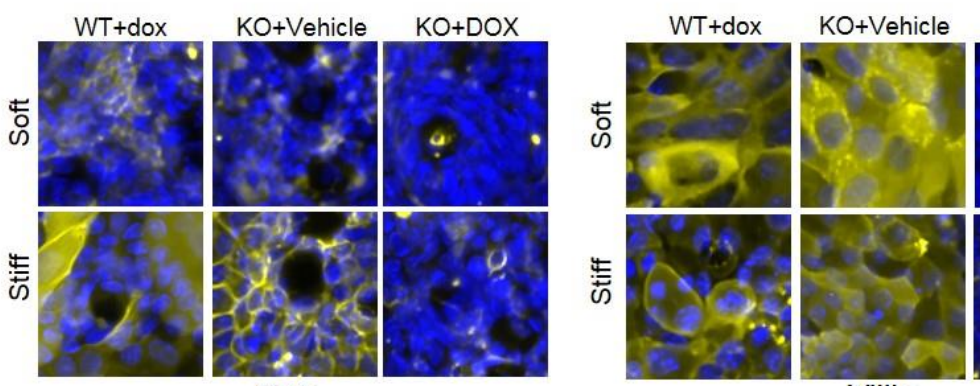

$\mathrm{KO}+\mathrm{DOX}$
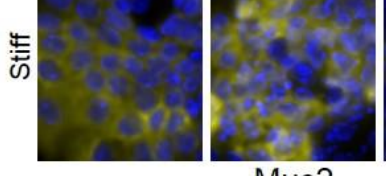

Egfr

Extended Data Figure 12. Staining for WT+DOX, YAP KO+Vehicle, and YAP KO+DOX on both soft The leftover crypt-like regions were enriched with Paneth cells and were negative for nuc-YAP and Muc2, 

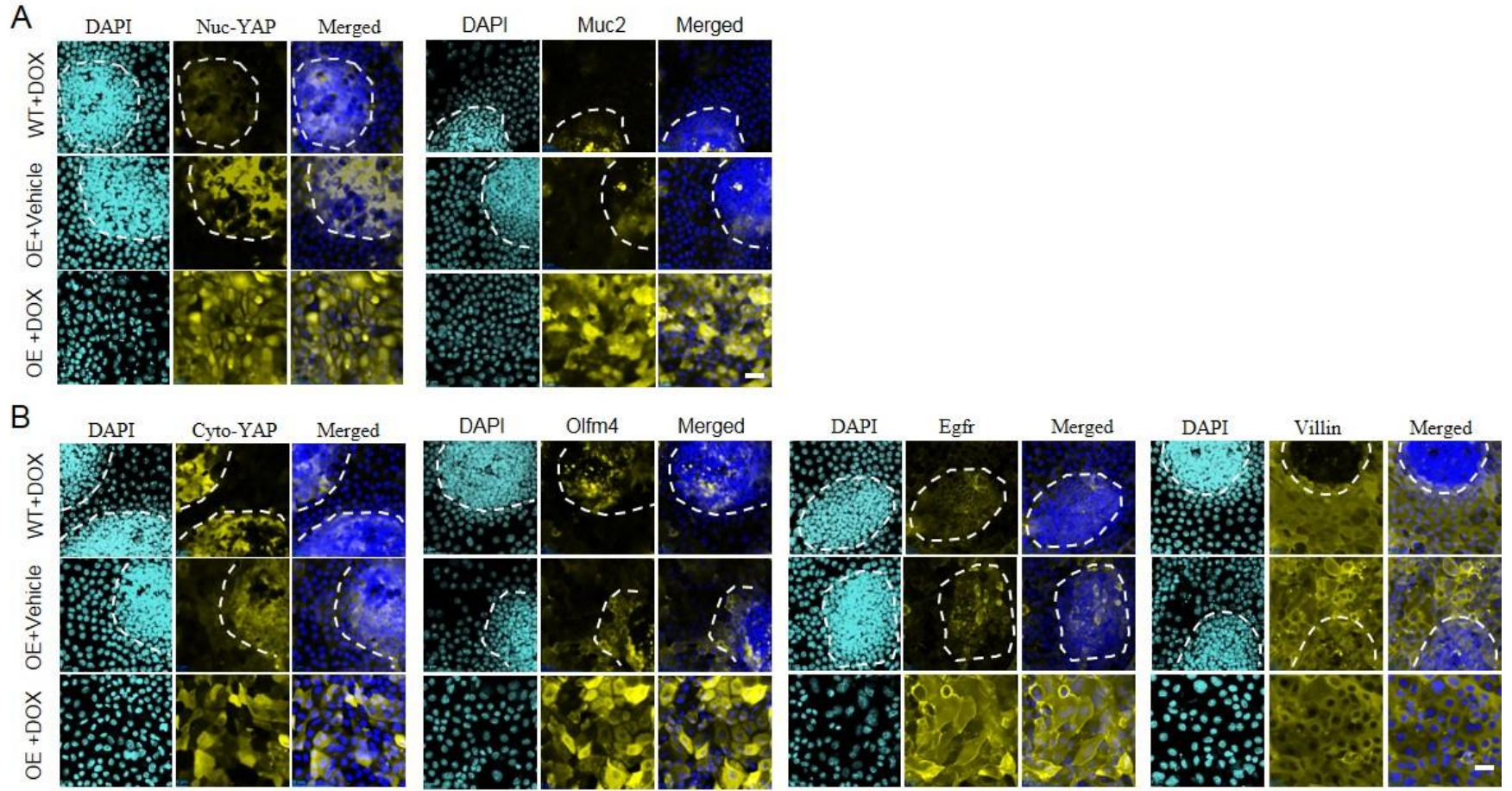

Extended Data Figure 13. Staining for WT+DOX, YAP OE+Vehicle, and YAP OE+DOX. (A) Increasing nuc-YAP expression by OE promoted Muc2. (B) The increase in cyto-YAP augmented the expression of Olfm4 and Egfr. The white dashed lines trace the crypt-like regions. $n=3$. Scale bar, $25 \mu \mathrm{m}$.

696 

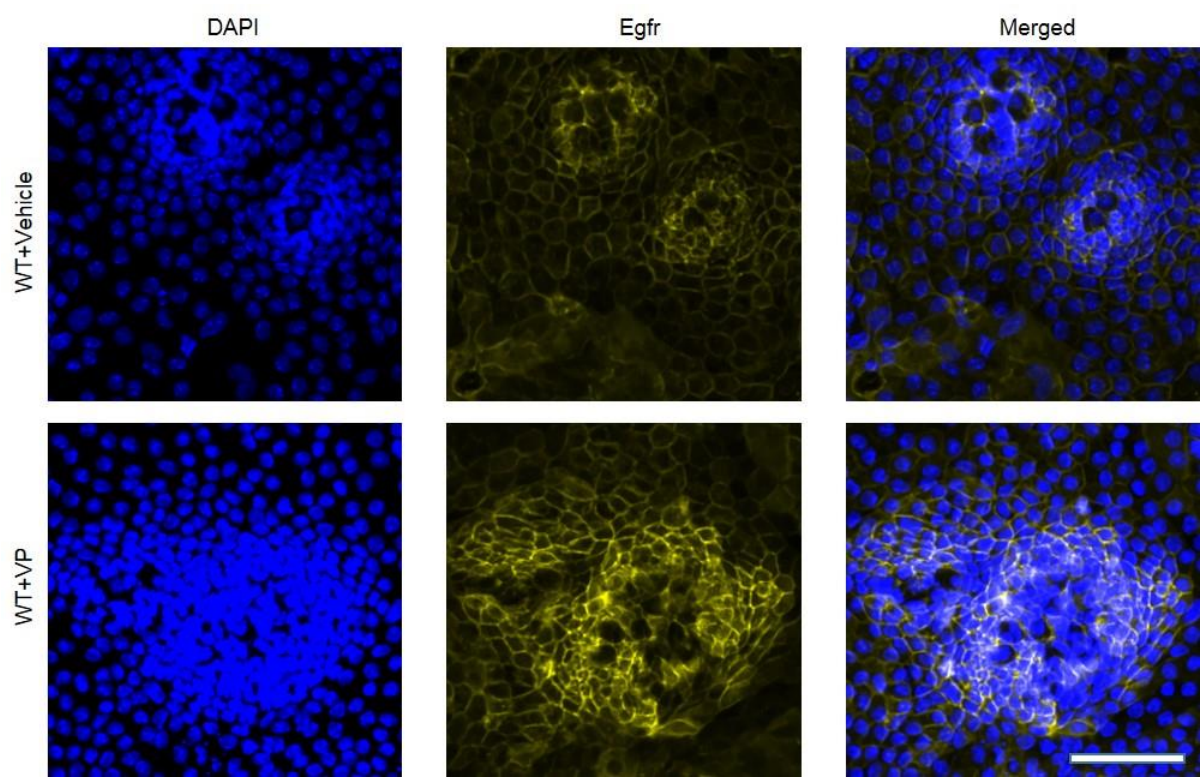

699 Extended Data Figure 14. Staining for WT+Vehicle and WT+VP. VP augmented the expression of 700 Egfr. $n=3$. Scale bar, $50 \mu \mathrm{m}$. 
A
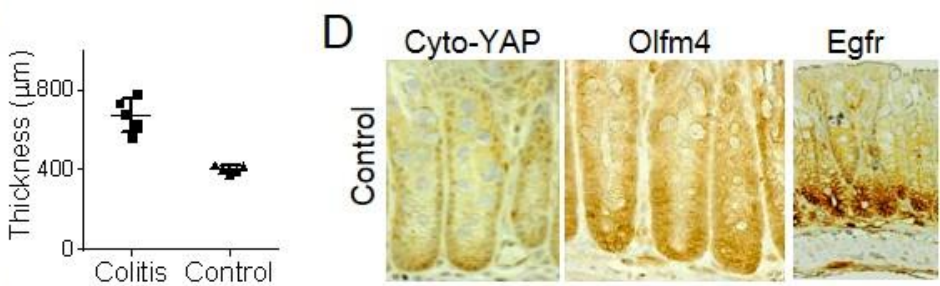

B
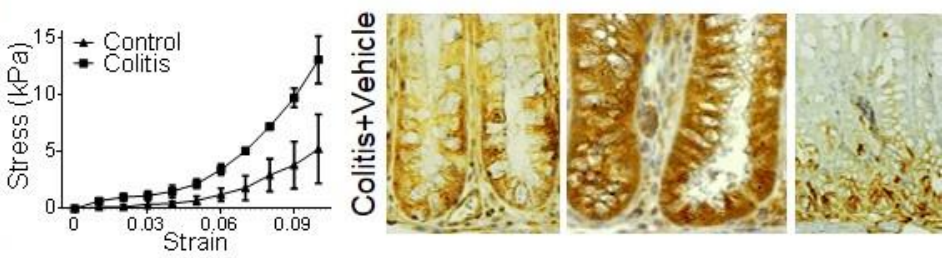

702
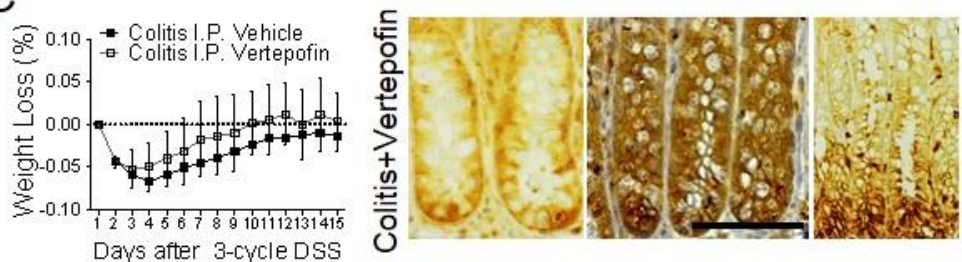

703 Extended Data Figure 15. Colon thickened (A, $n=6)$ and stiffened (B, $n=3)$ in colitis group compared to 704 the control. (C) VP treatment mitigated the body weight loss of the colitis group (n=6). (D) Cyto-YAP and 705 Olfm4 were lower in control than the other two groups. VP treatment significantly increased the 706 expression of Egfr. Scale bar, $200 \mu \mathrm{m}$.

707 
bioRxiv preprint doi: https://doi.org/10.1101/2021.03.15.435410; this version posted March 16, 2021. The copyright holder for this preprint (which was not certified by peer review) is the author/funder. All rights reserved. No reuse allowed without permission.

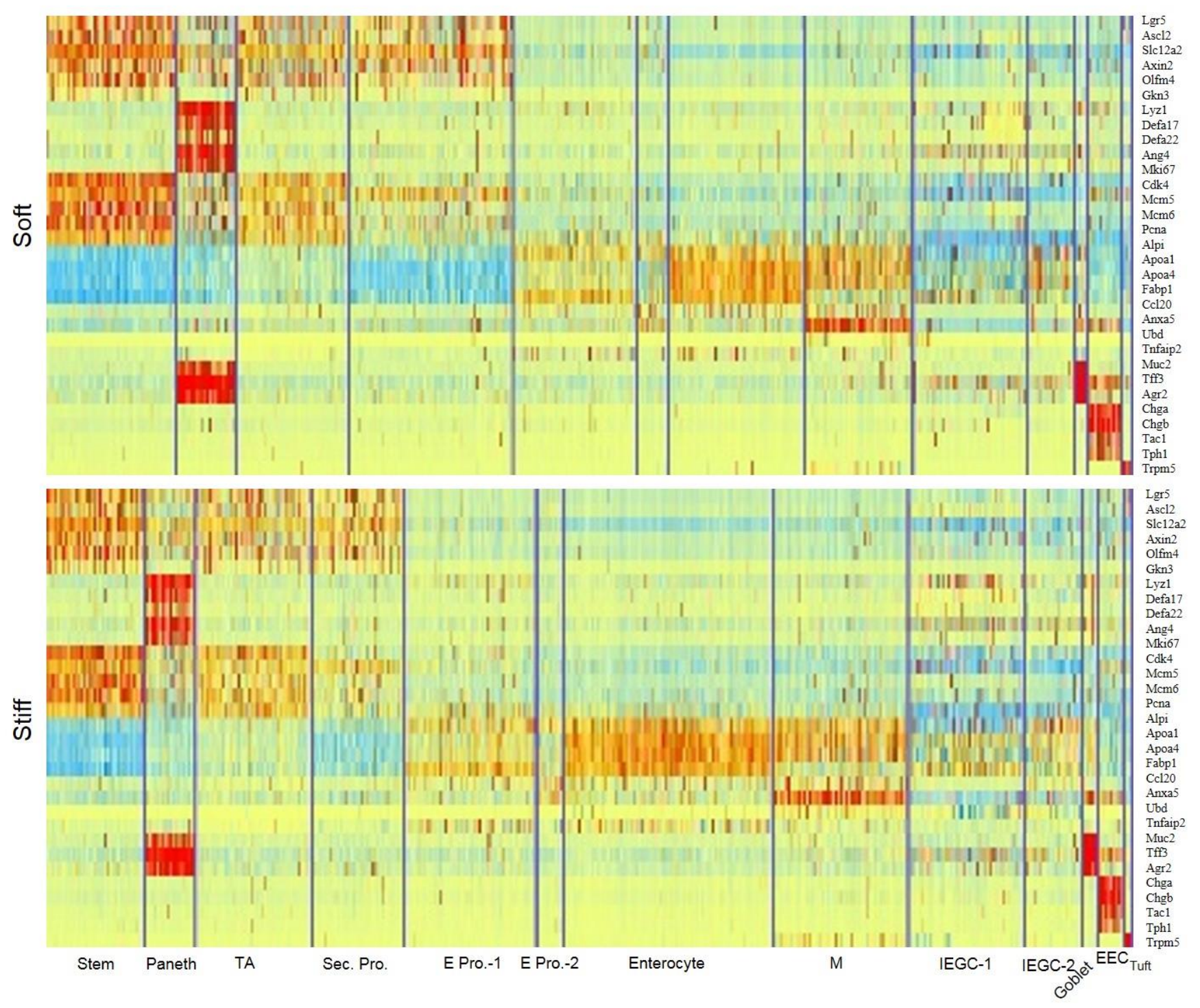

Extended Data Figure 16. Full labels of marker genes for each cell type. 
bioRxiv preprint doi: https://doi.org/10.1101/2021.03.15.435410; this version posted March 16, 2021. The copyright holder for this preprint (which was not certified by peer review) is the author/funder. All rights reserved. No reuse allowed without permission.

712

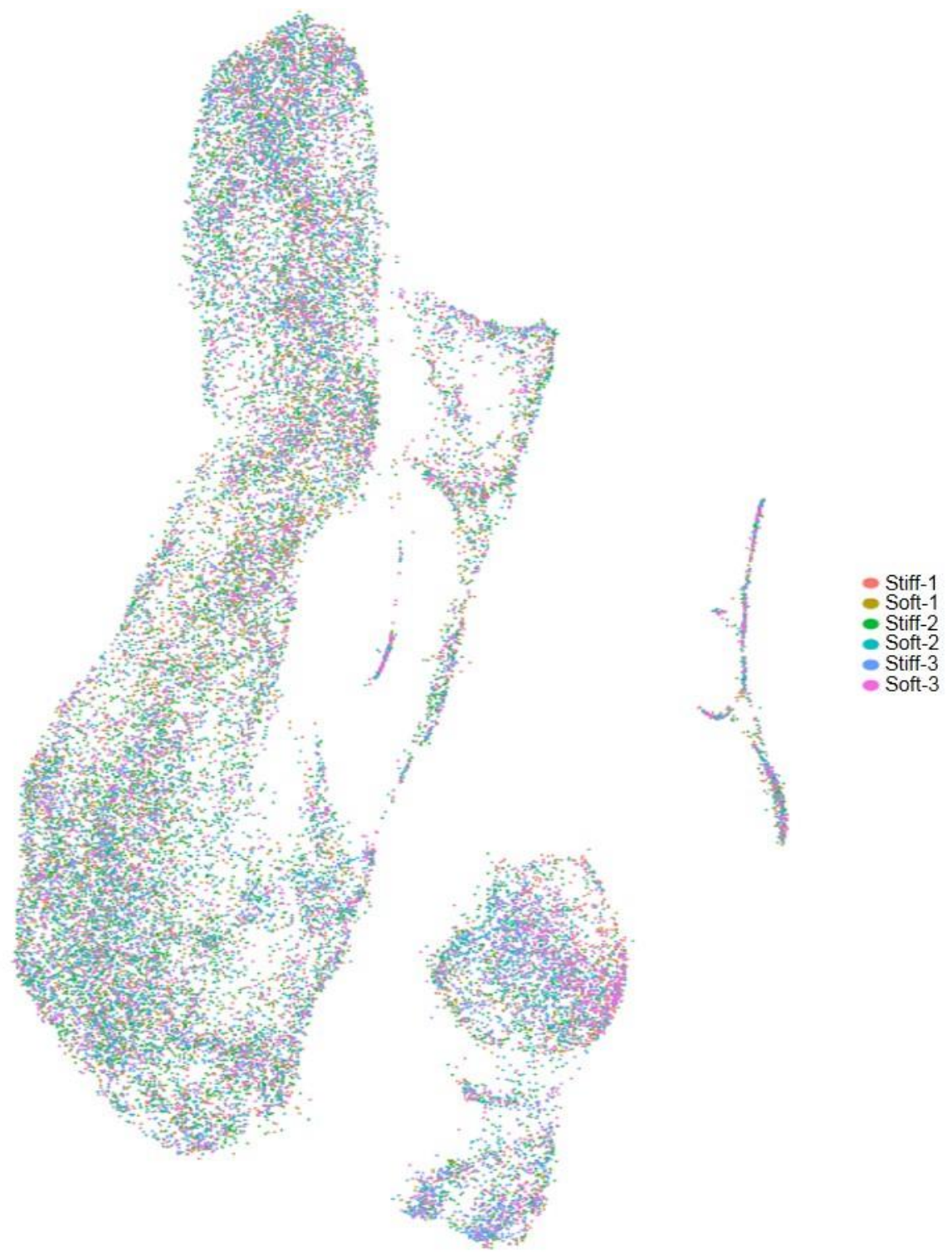

713

714

715

716

717
Extended Data Figure 17. Three animals were used to triplicate the single-cell expression profiles. The clustering was consistent among the triplication on both the soft matrix and the stiff martix. 

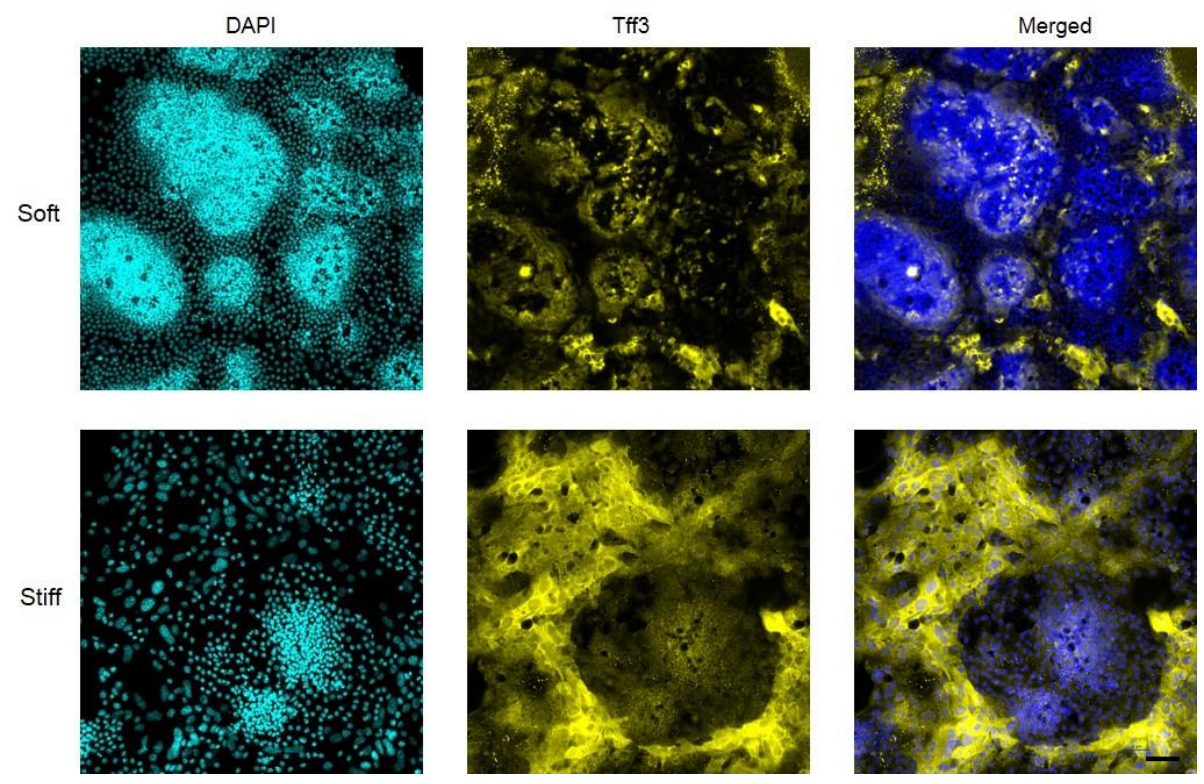

719 Extended Data Figure 18. The goblet cell marker- Tff3 was increased on the stiff matrix $(n=3)$. Scale 720 bar, $25 \mu \mathrm{m}$. 
YAP-downregulated Genes

723 Extended Data Figure 19. The genes downregulated by YAP highly expressed in enterocytes and their 724 progenitors-1 $(n=3)$. 


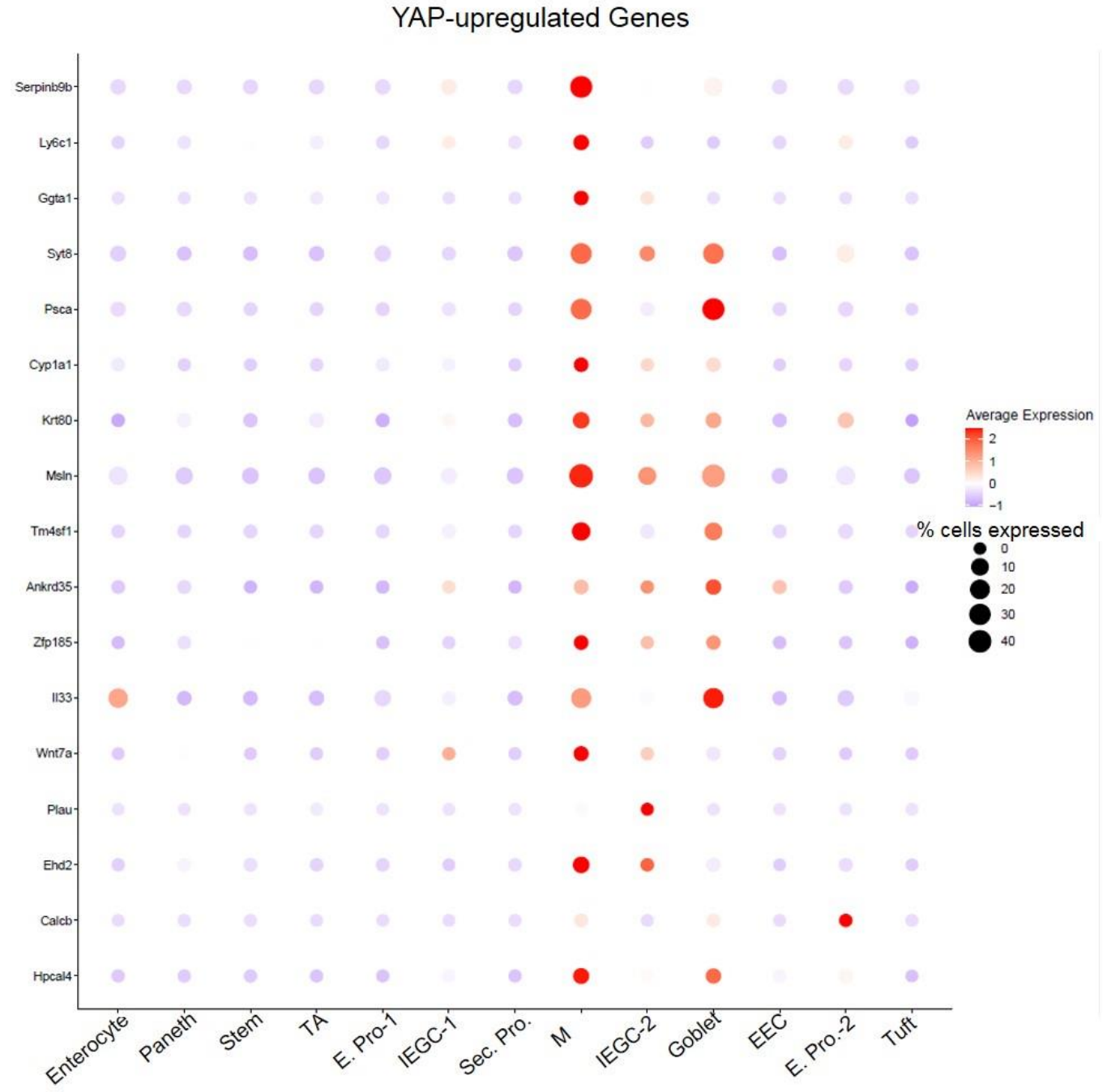

727 Extended Data Figure 20. The genes upregulated by YAP highly expressed in goblet cells, IEGCs and 728 M cells $(n=3)$. 
bioRxiv preprint doi: https://doi.org/10.1101/2021.03.15.435410; this version posted March 16, 2021. The copyright holder for this preprint (which was not certified by peer review) is the author/funder. All rights reserved. No reuse allowed without permission.

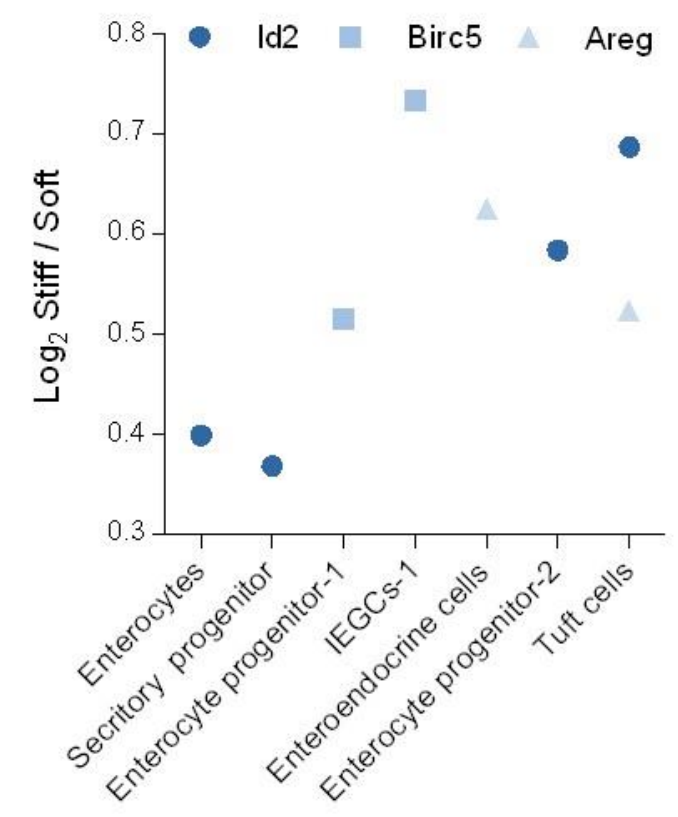

730

731 Extended Data Figure 21. Differential gene expressions analysis shows the changes of downstream 732 genes of nuc-YAP $(n=3)$.

733 
Galactose metabolism

Starch and sucrose metabolism

Lipid metabolism

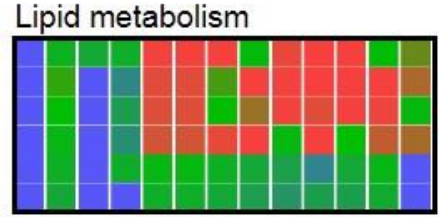

Glycerolipid metabolism

Fatty acid metabolism

Biosynthesis of unsaturated fatty acids

Fatty acid degradation

Primary bile acid biosynthesis

alpha-Linolenic acid metabolism
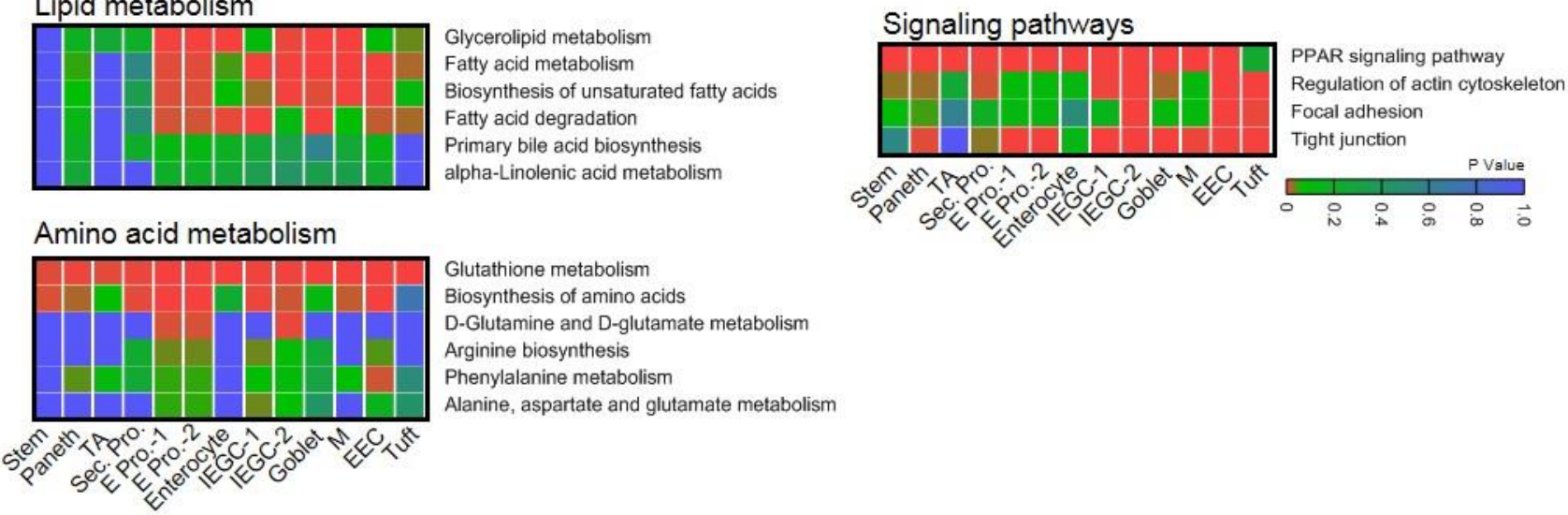

Extended Data Figure 22. Pathway enrichment analysis is performed for carbon metabolism, lipid

Glutathione metabolism

Biosynthesis of amino acids

D-Glutamine and D-glutamate metabolism

Arginine biosynthesis

Phenylalanine metabolism

Alanine, aspartate and glutamate metabolism

metabolism, amino acid metabolism and the signaling pathways. Compared to the soft matrix, carbon metabolism is more enriched than amino acid metabolism on the stiff matrix. Mechenotransductionrelated signaling as well as Peroxisome proliferator-activated receptor (PPAR) was also more enriched on the stiff matrix $(n=3)$. 
A

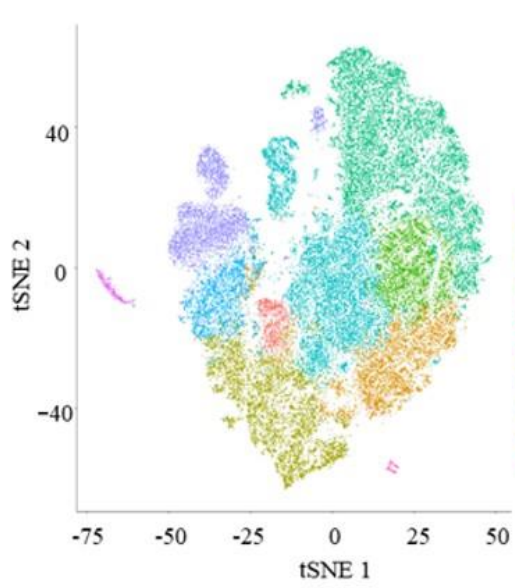

$\mathrm{C}$

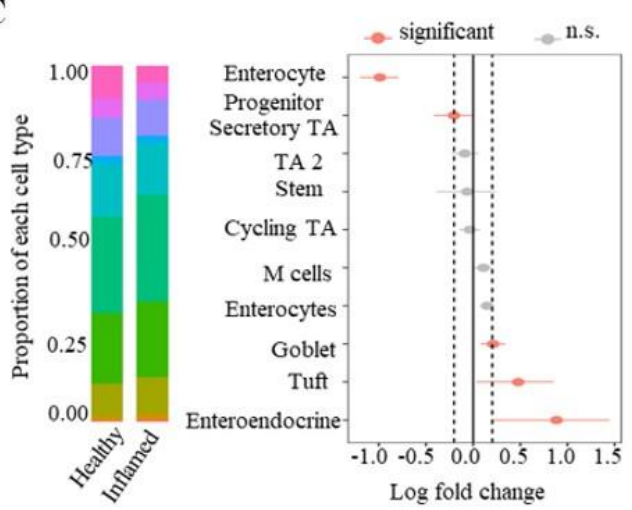

- Stem

- Cycling TA

M cells

Goblet
B

$\mathrm{D}$

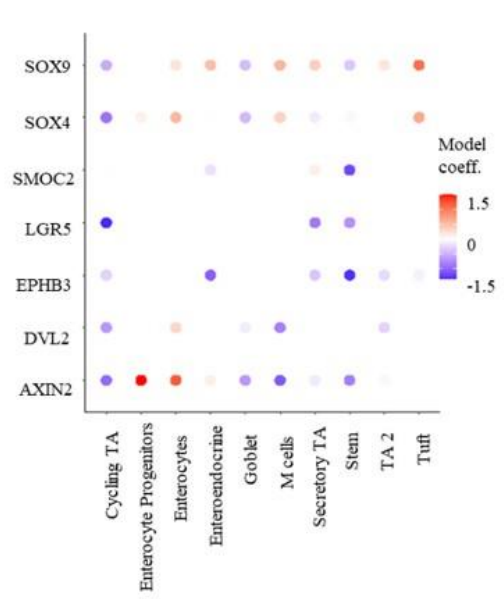

$\log _{2}(\mathrm{TP} 10 \mathrm{~K}+1)$ z-score $\begin{array}{lll}-2 & 0 & 2\end{array}$$$
\text { . }
$$

$\mathrm{E}$

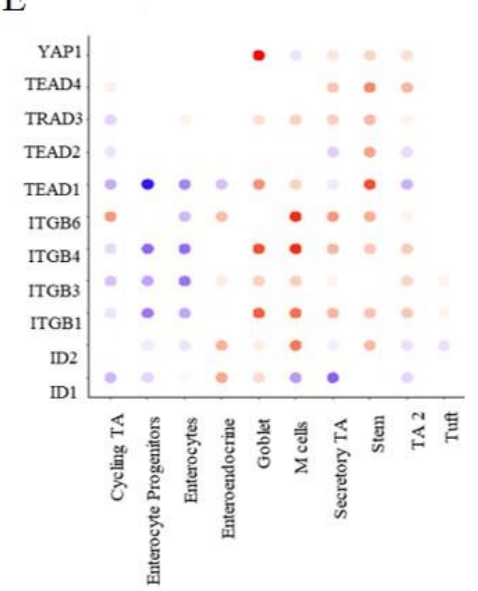

- Enterocyte Progen

- Enteroendocrine

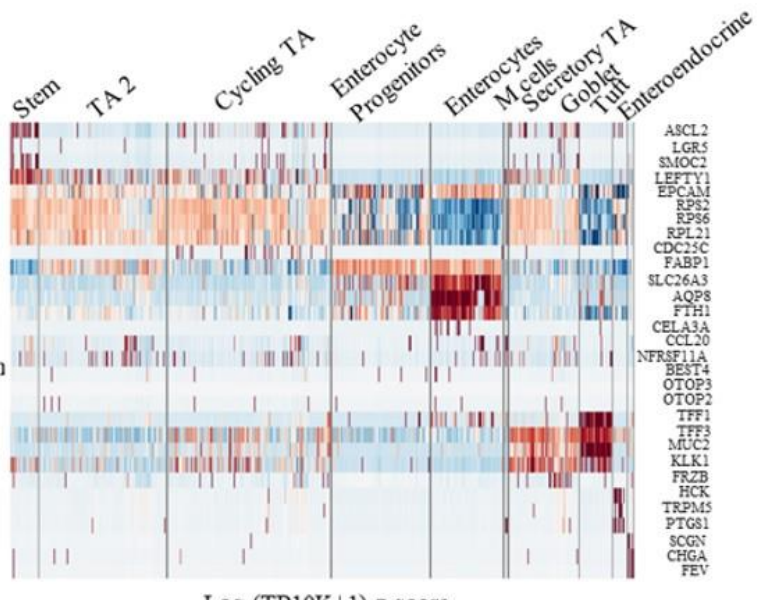



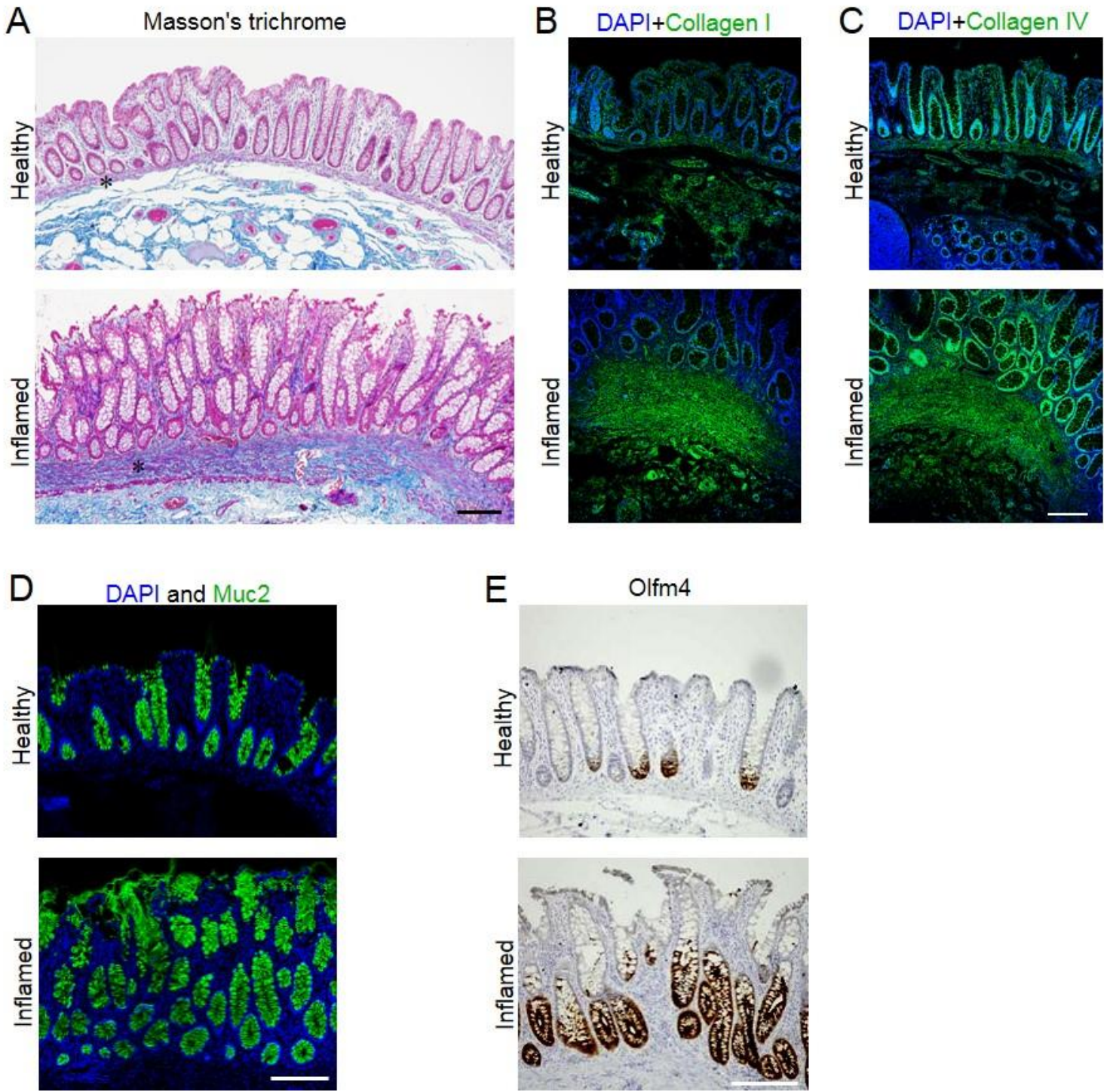

754 Extended Data Figure 24. The masson's trichorme staining (A) and the staining of collagen I (B) and 755 Collagen IV (C) showed the fibrosis and thickening of BM and lamina propria labelled with asterisks. (D) 756 Muc2 $^{+}$goblet cells increased in the inflamed colon. (E) Olfm4 was increased and expanded into larger 757 regions in the inflamed colon. $n=3$. Scale bar, $200 \mu \mathrm{m}$. 


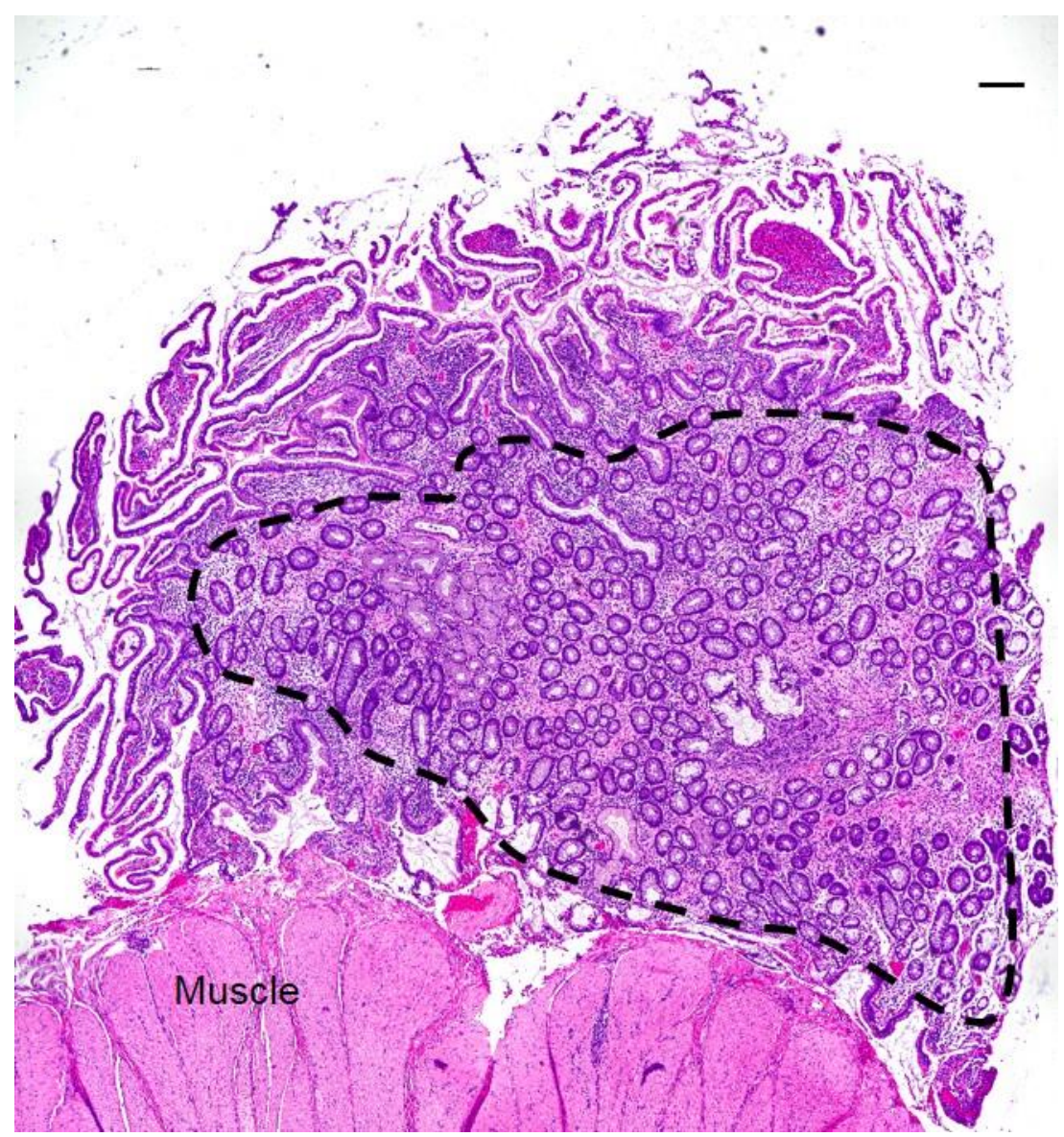

761 Extended Data Figure 25. In a large area (outside of the dashed line) of structured ileum (extreme 762 fibrosis), the invaginated ISC niche- crypts nearly disappeared and only pieces of the villi were left, 763 resembling the stiffness-reduced size of the crypt and loss of ISCs. Meanwhile, lots of ectopic crypts 764 (inside the dashed line) formed, resembling stiffness-induced new crypt formation. $n=1$. Scale bar, 200 765 $\mu \mathrm{m}$. 


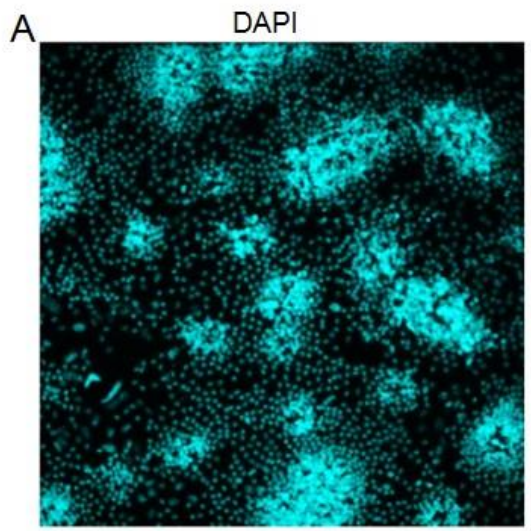

$\mathrm{B}$

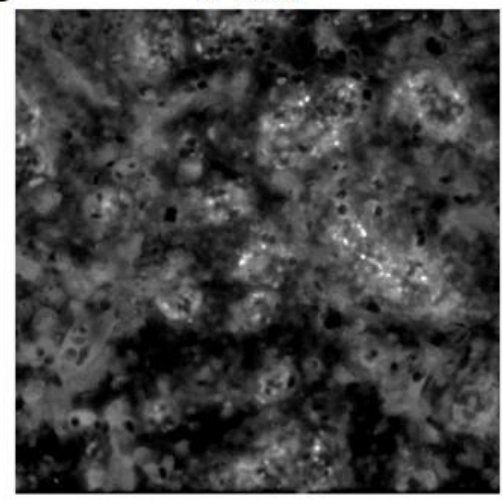

Identify the crypt-like regions

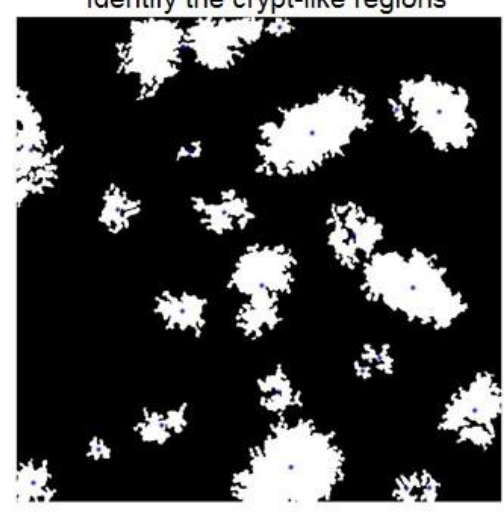

IF stain in the crypt-like regions

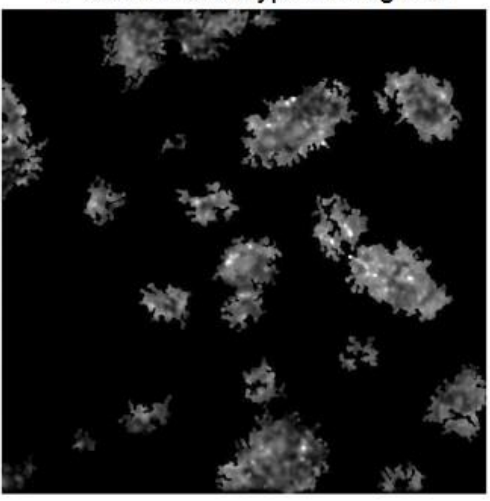

IF stain in the villus-like regions

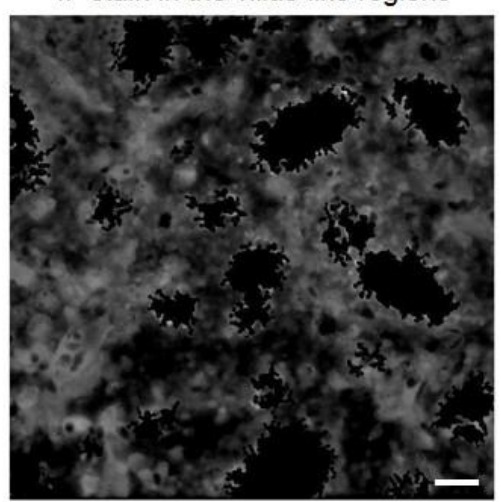

768 Extended Data Figure 26. Illustration for the customized MATLAB code. (A) The crypt-like regions

769 were identified based on the intensity of the DAPI staining. (B) The fluorescent signals were respectively

770 isolated in the crypt-like regions and the villus-like regions. Scale bar, $100 \mu \mathrm{m}$. 
A

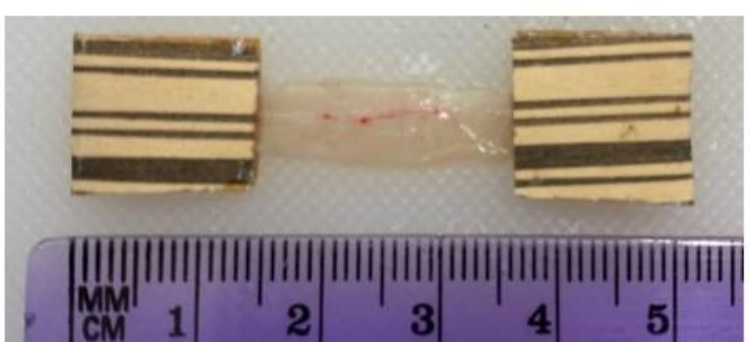

B

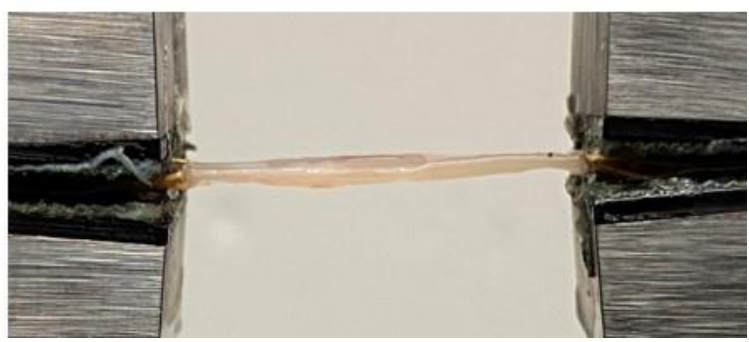

774 Extended Data Figure 27. (A) An intestinal tissue sample with sandpaper tabs at both ends; and (B) 775 uniaxial tensile test of the intestinal tissue sample. 